\title{
POLARS AND THEIR APPLICATIONS IN DIRECTED INTERPOLATION GROUPS $\left({ }^{1}\right)$
}

BY

\author{
A. M. W. GLASS
}

\begin{abstract}
In the study of $l$-groups, as in many other branches of mathematics, use is made of the concept of "orthogonal elements". The purpose of this paper is to show that this concept can be extended to directed, interpolation groups and that most of the theorems in $l$-groups concerning polars hold in the more general setting of directed, interpolation groups. As consequences, generalisations of Holland's and Lorenzen's theorems are obtained and a result on $o$-simple abelian, directed, interpolation groups.
\end{abstract}

1. Definitions, notation and basic facts. Let $S$ be a partially ordered set. $S$ will be said to satisfy the interpolation property if and only if whenever $s, t, u, v \in S$ and $s, t \leqq u, v$, then there exists $x \in S$ (not necessarily unique) such that $s, t \leqq x \leqq u, v$. If, in addition, $s \vee t$ and $s \wedge t$ do not exist in $S$ unless $s \leqq t$ or $t \leqq s$, then $S$ will be called an antilattice set.

Let $S$ be a partially ordered set and $X \subseteq S$. Then $\mathscr{L}(X)$, the set of lower bounds of $X$ in $S$, will denote $\{s \in S: s \leqq x$ for all $x \in X\}$ and $\mathscr{U}(X)$, the set of upper bounds of $X$ in $S$, will denote $\{s \in S: s \geqq x$ for all $x \in X\}$. In the special case that $X$ is finite, say $X=\left\{x_{1}, \ldots, x_{n}\right\}$, we will write $\mathscr{L}\left(x_{1}, \ldots, x_{n}\right)$ for $\mathscr{L}(X)$ and $\mathscr{U}\left(x_{1}, \ldots, x_{n}\right)$ for $\mathscr{U}(X)$. If $s, t \in S, s \$ t$ and $t \$ s$, then we will write $s \| t$. $\varnothing$ will denote the empty set, $\subset$ will denote strict containment and $\subseteq$ will denote weak containment.

Throughout this paper, additive notation will be used for the group operation (it is not to be assumed that the group is abelian). If $G$ is a group and $X \subseteq G$, then $\langle X\rangle$ will denote the subgroup of $G$ generated by $X$.

$G$ is an interpolation group if and only if $G$ is a p.o. group (partially ordered group) whose partial order satisfies the interpolation property. It is easy to see that $G$ is an interpolation group iff whenever $0 \leqq g \leqq h_{1}+h_{2}$ and $h_{i} \geqq 0(i=1,2)$, then there exist $g_{1}, g_{2} \in G$ such that $0 \leqq g_{i} \leqq h_{i}(i=1,2)$ and $g=g_{1}+g_{2}$.

If $G$ is a p.o. group whose partial order is an antilattice set, then $G$ is said to be an antilattice. If $G$ is an interpolation group, then $\mathscr{C}(G)$ will denote the complete

Received by the editors March 25, 1971.

AMS 1970 subject classifications. Primary 06A55.

Key words and phrases. Carrier, convex directed subgroup, directed, interpolation property, antilattice, polar, prime subgroup, prime filter, bipolar, representable directed, interpolation group.

(1) This paper represents a portion of the author's Ph.D. thesis at the University of Wisconsin, under the direction of Professor W. Charles Holland, to whom the author wishes to express gratitude. 
lattice of convex $d$-subgroups (convex directed subgroups) of $G$ (see [11]). In the special case that $\mathscr{C}(G)$ is closed under set-theoretic intersection, $G$ is said to be a strong interpolation group. $M \in \mathscr{C}(G)$ is said to be a value of $g \in G$ iff $g \notin M$ and $M \subset N \in \mathscr{C}(G)$ implies that $g \in N$.

If $G$ is a p.o. group and $S \subseteq G$, then $S^{+}$denotes $\{s \in S: s \geqq 0\}$ and $S^{-}$denotes $\{s \in S: s \leqq 0\}$. If $C$ is a convex subgroup, then $\mu(C)=\left\langle C^{+}\right\rangle$is the largest convex $d$-subgroup of $G$ contained in $C$ (see [11]). If $n g \geqq 0$ for some positive integer $n$ implies $g \geqq 0$, then $G$ is said to be semi-isolated, and if $n g>0$ for some positive integer $n$ implies $g>0$, then $G$ is said to be weakly semi-isolated.

If $A$ and $B$ are p.o. groups, then $A \boxplus B$, the cardinal sum of $A$ and $B$, denotes the group $A \oplus B$ ordered by: $(a, b) \geqq 0$ iff $a \geqq 0$ (in $A$ ) and $b \geqq 0$ (in $B$ ) and $A \oplus \rightarrow B$, the lexicographic sum of $A$ over $B$, denotes the group $A \oplus B$ ordered by: $(a, b) \geqq 0$ iff $a>0$ (in $A$ ) or ( $a=0$ and $b \geqq 0$ (in $B$ )).

$\boldsymbol{Z}(\boldsymbol{R})$ will denote the totally ordered additive group of integers (real numbers) but $\boldsymbol{Z}^{+}\left(\boldsymbol{R}^{+}\right)$will denote $\{m \in \boldsymbol{Z}: m>0\}(\{r \in \boldsymbol{R}: r>0\})$.

Let $A$ be a p.o. set and let $\left\{G_{\alpha}: \alpha \in A\right\}$ be a family of semi-isolated abelian groups. Let $K=\prod\left\{G_{\alpha}: \alpha \in A\right\}$ and $S(k)=\left\{\alpha \in A: k_{\alpha} \neq 0\right\}$ for $k \in K$. Let $V=V\left(A, G_{\alpha}\right)$ be the group $\{k \in K: S(k)$ contains no infinite ascending sequence in $A\}$. For each $v \in V$, let $M(v)=\left\{\alpha \in A: v_{\alpha} \neq 0\right.$ and $v_{\beta}=0$ for all $\left.\beta>\alpha\right\}$. Define $v>0$ iff $v_{\alpha}>0$ for all $\alpha \in M(v)$. Then, with this partial ordering, $V$ is an abelian, semi-isolated group (see [6]). $W=\Sigma\left(A, G_{\alpha}\right)=\{v \in V: S(v)$ is finite $\}$ is an abelian, semi-isolated subgroup of $V$.

Suppose $G$ is an $l$-group (lattice-ordered group). For each $g \in G$, write $|g|$ for $g \vee-g . g, h \in G$ are said to be orthogonal if and only if $|g| \wedge|h|=0$. The polar of $g$, $p(g)$, is defined to be the set of all $h \in G$ which are orthogonal to $g$. If $S \subseteq G$, then the polar of $S, p(S)=\bigcap\{p(s): s \in S\}$. It is easy to verify that polars are convex l-subgroups of $G$.

A generalization of the concept of polar is obtained by taking any convex $l$-subgroup $C$ of the $l$-group $G$ and defining $p(g, C)$, the $C$-polar of $g$, to be $\{x \in G:|x| \wedge|g| \in C\}$ and $p(S, C)$, the $C$-polar of $S$, to be $\bigcap\{p(s, C): s \in S\}$ where $g \in G$ and $S \subseteq G$. This definition is indeed a generalization since the polar of $S$ is precisely the $\{0\}$-polar of $S$. It is due to R. D. Byrd (see [2] or [3]). $C$-polars are convex $l$-subgroups of the $l$-group $G$ and contain $C$.

If $G$ is an $l$-group, then $z \leqq|g|$ iff $z \in \mathscr{L}(\mathscr{U}(g, 0,-g))$; therefore, $x \in p(g)$ iff $\mathscr{L}(\mathscr{U}(g, 0,-g)) \cap \mathscr{L}(\mathscr{U}(x, 0,-x)) \subseteq \mathscr{L}(0)$.

2. Definition of $C$-polars in directed, interpolation groups. Let $G$ be a p.o. group and $f \in G^{+}$. Let $f^{\perp}=\left\{h \in G^{+}: h \wedge f=0\right\}$. $P$, a map from $G$ into the set of subsets of $G$, is said to be a concept of polar if and only if

(1) $P(g)$ is a convex subgroup of $G$ for all $g \in G$,

(2) if $g \in G^{+}$, then $P(g)=\left\langle g^{\perp}\right\rangle$, and

(3) if $g, h \in G$, then $g \in P(h)$ iff $h \in P(g)$. 
For the remainder of this paper it will be assumed that $G$ is a directed, interpolation group and $C \in \mathscr{C}(G)$.

Lemma 1. If $g \in G^{+}$, then $X(g, C)=\left\{h \in G^{+}:\right.$for all $f \in G$, if $f \leqq g, h$, then $f \leqq c_{f}$ for some $\left.c_{f} \in C\right\}$ is a convex subsemigroup of $G^{+}$containing $C^{+}$(and hence 0 ). If $C=\{0\}$, then $X(g, C)=g^{\perp}$.

Proof. Suppose $a, b \in X(g, C)$ and that $x \leqq a+b, g$; so there exists $y \in G$ such that $0, x \leqq y \leqq a+b, g$. But $0 \leqq a, b$. Hence there exist $y_{1}, y_{2} \in G^{+}$such that $y_{1} \leqq a$, and $y_{2} \leqq b$, and $y=y_{1}+y_{2}$ ( $G$ is an interpolation group). Now $y_{1}, y_{2} \leqq y \leqq g$. Hence $y_{1} \leqq a, g$ and $y_{2} \leqq b, g$. Thus there exist $c, d \in C$ such that $y_{1} \leqq c$ and $y_{2} \leqq d$. Therefore $x \leqq y \leqq c+d \in C$. Consequently, $a+b \in X(g, C)$ and $X(g, C)$ is a subsemigroup of $G^{+}$. If $0 \leqq h \leqq a$ and $a \in X(g, C)$, it is immediate that $h \in X(g, C)$; so $X(g, C)$ is convex. Finally, if $c \in C^{+}$and $x \leqq c, g$, then $x \leqq c$. Thus $c \in X(g, C)$ and $C^{+}$ $\subseteq X(g, C)$.

Let $g \in G^{+} \cdot p_{0}(g, C)=\langle X(g, C)\rangle$ and $p_{0}(g)=p_{0}(g,\{0\})$. If $g \in G^{-}$, then define $p_{0}(g, C)$ to be $p_{0}(-g, C)$ and $p_{0}(g)$ to be $p_{0}(-g)$. The following lemmas will lead to a definition of $C$-polars of elements unrelated to 0 .

Lemma 2. If $0 \leqq g \leqq h$, then $p_{0}(g, C) \supseteq p_{0}(h, C)$. Hence if $0 \geqq g \geqq h$, then $p_{0}(g, C)$ $\supseteq p_{0}(h, C)$, i.e. the further from 0 , the smaller the $C$-polar.

Proof. It is trivial to prove that if $0 \leqq g \leqq h$, then $X(h, C) \subseteq X(g, C)$. The result follows at once from this.

LEMMA 3. $p_{0}(g, C)=\bigcup\left\{p_{0}(h, C): h \in G\right.$ such that $\left.h \geqq g, 0,-g\right\}$ for all $g \in G^{+}$ $\cup G^{-}$.

The proof is easy and so is omitted.

Now define the $C$-polar of $g \in G, p(g, C)$, to be $\bigcup\left\{p_{0}(h, C): h \in G\right.$ such that $h \geqq g, 0,-g\}$. If $g \in G^{+} \cup G^{-}$, then $p(g, C)=p_{0}(g, C)$ by Lemma 3. The polar of $g \in G, p(g)$, is defined to be $p(g,\{0\})$.

TheOREM 4. For all $g \in G, p(g, C) \in \mathscr{C}(G)$ and $C \subseteq p(g, C)$. If $P$ is a concept of polar, then for all $g \in G, p(g) \subseteq P(g)$ and $p$ is the unique concept of polar such that $p(g) \in \mathscr{C}(G)$ for all $g \in G$.

Proof. If $g$ is related to 0 , then $p(g, C) \in \mathscr{C}(G)$ and $C \subseteq p(g, C)$ by Lemma 3 and the remarks following Lemma 1 . Now for all $g \in G,\{p(h, C): h \geqq g, 0,-g\}$ is an upper directed set under set-theoretic inclusion because of the interpolation property and Lemma 2 . Hence $C \subseteq p(g, C) \in \mathscr{C}(G)$ for all $g \in G$ follows from the special case when $g \in G^{+}$.

Suppose $g \in p(h, C)$. Then $g \in p(k, C)$ for some $k \geqq h, 0,-h . p(k, C) \in \mathscr{C}(G)$, so there exist $x, y \in p(k, C)^{+}$such that $g=x-y$ and there exists $z \in p(k, C)^{+}$ such that $z \geqq x, y$. Hence $z \geqq g, 0,-g$ and it follows from the definition that $p(z, C) \subseteq p(g, C)$. By definition, $k \in p(z, C)^{+}$(since $k, z \geqq 0, X(z, C)=p(z, C)^{+}$ 
and $\left.X(k, C)=p(k, C)^{+}\right)$, so $k \in p(g, C)$. But $-k \leqq h \leqq k$ and $p(g, C)$ is convex. Therefore, $h \in p(g, C)$. It now follows that $p$ is a concept of polar.

If $P$ is a concept of polar and $g \in G^{+}$, then $P(g)=\left\langle g^{\perp}\right\rangle=p(g)$, and if $f \in G$ and $0 \leqq k \in p(f)$, then $f \in p(k)=\left\langle k^{\perp}\right\rangle=P(k)$. It follows that $k \in P(f)$ so $p(f) \subseteq P(f)$ for all $f \in G$ and, similarly, $P(f)^{+} \subseteq p(f)^{+}$for all $f \in G$. If $P(g)$ is directed for all $g \in G$, then $P(f)=p(f)$ for all $f \in G$.

Corollary 4.1. For all $g, h \in G, g \in p(h, C)$ iff $h \in p(g, C)$.

COROLLARY 4.2. If $G$ is an l-group then the definition given for l-groups coincides with the one given here.

Proof. This follows from the fact that if $G$ is an l-group, then $C$ is a convex $l$-group of $G$ if and only if $C$ is a convex directed subgroup of $G$.

An alternative possible definition of the polar of $g \in G$ is given by

$$
p_{1}(g)=\{x \in G: \mathscr{L}(\mathscr{U}(g, 0,-g)) \cap \mathscr{L}(\mathscr{U}(x, 0,-x)) \subseteq \mathscr{L}(0)\} .
$$

As has already been noted, this definition agrees with that for $l$-groups in the case that $G$ is an $l$-group. However, $p_{1}$ is not a concept of polar as property (2) need not hold (see, e.g., $\boldsymbol{R} \oplus \boldsymbol{R}$ ordered by: $(x, y)>0$ iff $x>0$ and $y \geqq 0$ ). If $G$ is even (i.e., every $g \in G$ can be expressed as $2 h$ for some $h \in G$ ) and strongly semi-isolated (no sum of conjugates of an element $g \geq 0$ is $\geqq 0$ ), then it can be shown that $p_{1}$ satisfies properties (1) and (3) of the definition of a concept of polar.

If $G$ is an antilattice, then $p(g)=\{0\}$ for all $g>0$ and hence $p(g)=\{0\}$ for all $g \in G \backslash\{0\}$. Consequently, the polars in an antilattice are trivial ( $\{0\}$ or $G)$. This is in contrast to $p_{1}$ as is seen from the example mentioned above. In the case that $S$ is a subset of $G$, the $C$-polar of $S, p(S, C)$, can be defined to be $\Pi\{p(s, C): s \in S\}$ $=\mu(\bigcap\{p(s, C): s \in S\})$. It now follows that $C$-polars (and so polars, which are $\{0\}$-polars) are convex $d$-subgroups which contain $C$. Observe that $p(g, C)$ $=p(\{g\}, C)$.

The definition can be extended to $K$-polars, where $K$ is a convex subgroup (not necessarily directed) of $G$. However, if the definition is given with $K$ in place of $C$, it is easy to see that $p(S, K)=p(S, \mu(K))$. Hence nothing is gained by this extra generalization.

3. Properties of $C$-polars. In this section, proofs of results will often be omitted since they can be obtained by adapting those for $l$-groups (to be found in [2] or [3]). Some proofs will be given as samples. It cannot be stressed too much that extreme care must be taken in the adaptation. Although most of the results which hold for polars in l-groups are true for polars in directed, interpolation groups, not all results carry over. Examples will be given in cases where the analogue fails.

Lemma 5. (i) If $x, y \in G^{+}$, then $p(x, C) \cap p(y, C)=p(x+y, C)$.

(ii) If $S$ and $T$ are subsets of $G$ and $S \subseteq T$, then $p(T, C) \subseteq p(S, C)$. 
Proof. We prove that $p(x, C)^{+} \cap p(y, C)^{+} \subseteq p(x+y, C)^{+}$. The rest of the proof is elementary. Suppose $z \in p(x, C)^{+} \cap p(y, C)^{+}$and $0 \leqq t \leqq z, x+y$. As $G$ is an interpolation group, there exist $u, v \in G^{+}$such that $u+v=t$ and $u \leqq x$ and $v \leqq y$. Now $0 \leqq u \leqq z, x$ and $0 \leqq v \leqq z, y$. Hence $u, v \in C$. Thus $t \in C$ and the proof is completed.

LEMMA 6. (i) If $S$ is a subset of $G$, then the following are equivalent:

(a) $S \subseteq C$.

(b) $p(S, C)=G$.

(c) $S \subseteq p(S, C)$.

(ii) For all $g \in G, p(g, C)=G$ if and only if $g \in C$.

Proof. We prove (c) $\rightarrow$ (a). Assume $S \subseteq p(S, C)$. Then $s \in p(S, C) \subseteq p(s, C)$ for all $s \in S$. Consequently, there exists $g \geqq s, 0,-s$ such that $s \in p(g, C)$. But $p(g, C)$ is directed. It follows that there exists $h \in p(g, C)$ such that $h \geqq s, 0,-s$. Now $s,-s \leqq h, g$ and $h \in X(g, C)$. Hence there exists $c_{1}, c_{2} \in C$ such that $s \leqq c_{1}$ and $-s \leqq c_{2}$. Therefore, $-c_{2} \leqq s \leqq c_{1}$ and so $s \in C$. Thus $S \subseteq C$.

LeMmA 7. (i) If $L, M \in \mathscr{C}(G)$ and $L \subseteq M$, then $p(S, L) \subseteq p(S, M)$ for all $S \subseteq G$. In particular, if $A, B \in \mathscr{C}(G)$, then $p(S, A \cap B) \subseteq p(S, A) \cap p(S, B)$.

(ii) If $S \in \mathscr{C}(G)$, then $p(S, C)=\mu\left(\bigcap\left\{p(s, C): s \in S^{+}\right\}\right)$.

(iii) If $S, A, B \in \mathscr{C}(G)$, then $p(S, A) \cap p(S, B)=p(S, A \cap B)$. In fact, $p(g, A)$ $\cap p(g, B)=p(g, A \cap B)$ for all $g \in G^{+} \cup G^{-}$.

(iv) If $S \in \mathscr{C}(G)$, then $p(S, C)=p(S, S \cap C)$.

A definition of higher $C$-polars is now given using induction on the positive integer $n$. Let $S$ be a subset of $G \cdot p^{1}(S, C)=p(S, C)$ and $p^{n+1}(S, C)=p\left(p^{n}(S, C), C\right)$, the $(n+1)$ th $C$-polar of $S$. For all $n \in Z^{+}, C \subseteq p^{n}(S, C) \in \mathscr{C}(G)$ for each $S \subseteq G$. By Lemma 5(ii), if $S \subseteq T \subseteq G$, then $p^{2}(S, C) \subseteq p^{2}(T, C) \subseteq p^{2}(G, C)=G$ (this last fact follows from $p(G, C)=C$ and Lemma 6(i)).

Let $S$ be a subset of $G$. $S$ is said to be weakly positive if and only if for all $s \in S$, there exist $s_{1}, s_{2} \in S^{+} \cup S^{-}$such that $s_{1} \leqq s \leqq s_{2}$.

LeMma 8. (i) For every subset of $S$ of $G, S^{+} \cup S^{-} \subseteq p^{2}(S, C)$. If $S$ is a weakly positive set, then $S \subseteq p^{2}(S, C)$.

(ii) If $G$ is a strong interpolation, directed group, then $S \subseteq p^{2}(S, C)$ for every subset of $G$.

Proof. (i) It is immediate from Corollary 4.1 that $S \subseteq \bigcap\{p(x, C): x \in p(S, C)\}$ and so $S^{+} \cup S^{-} \subseteq p^{2}(S, C)$.

(ii) If $G$ is a directed, strong interpolation group, then $\bigcap\{p(x, C): x \in p(S, C)\}$ $=p^{2}(S, C)$.

Since every $l$-group is a strong interpolation, directed group, $S \subseteq p^{2}(S, C)$ for all $S \subseteq G$ holds for $l$-groups. A directed, interpolation group $G$ is said to be a strong $C$-polar group if and only if $S \subseteq p^{2}(S, C)$ for every subset $S$ of $G$. 
Let $A=Z^{-} \cup\{a\}$ where $a<\alpha$ for all $\alpha \in Z^{-}$and $Z^{-}$has the usual ordering. Let $R_{\alpha}=\boldsymbol{R}$ if $\alpha \in Z^{-}$and let $R_{a}$ be the totally unordered additive group of reals. Let $G=V\left(A, R_{\alpha}\right)$. Then $G$ is a strong $\{0\}$-polar group (if $S \neq \varnothing,\{0\}$, then $p(S)=\{0\}$ ) but not a strong interpolation group (see [9]).

Note that if $S \in \mathscr{C}(G)$, then $S$ is a weakly positive set as is every set of positive or negative elements of $G$.

Lemma 9. (i) $p(S, C) \cap p^{2}(S, C)=C$ for every subset $S$ of $G$.

(ii) If $C \subseteq S$ and $S$ is a weakly positive set, then $p(S, C) \cap S=C$.

(iii) If $C \subseteq S$ and $G$ is a strong interpolation, directed group, then $p(S, C) \cap S=C$.

(iv) If $C \subseteq S \in \mathscr{C}(G)$, then $p(S, C)$ is the largest convex $d$-subgroup of $G$ whose intersection with $S$ is contained in (or equal to) $C$.

Proof. (i) It is enough to show that if $0 \leqq g \in p(S, C) \cap p^{2}(S, C)$, then $g \in C$. This follows at once from Lemma 6(i).

(ii) and (iii) follow from the previous lemma.

(iv) Suppose $M \in \mathscr{C}(G)$ and $M \cap S \subseteq C$. Let $0 \leqq x \in M$ and $0 \leqq s \in S$. Then $x \in p(s, C)^{+}$. By Lemma 7(ii), $M \subseteq p(S, C)$.

LEMMA 10. (i) For every subset $S$ of $G, p(S, C) \subseteq p^{3}(S, C)$ and $p^{2}(S, C)=p^{4}(S, C)$.

(ii) If $S \subseteq p^{2}(S, C)$, then $p(S, C)=p^{3}(S, C)$.

(iii) If $S$ is a weakly positive set, $p(S, C)=p^{3}(S, C)$.

(iv) If $G$ is a strong interpolation, directed group, then $p(S, C)=p^{3}(S, C)$ for every subset of $G$.

Proof. (i) By Lemma $8(\mathrm{i}), p(S, C) \subseteq p^{3}(S, C)$, since $p(S, C) \in \mathscr{C}(G)$. Hence $p^{4}(S, C) \subseteq p^{2}(S, C)$. Using $p^{2}(S, C) \in \mathscr{C}(G), p^{2}(S, C) \subseteq p^{4}(S, C)$ by Lemma 8(i).

(ii) If $S \subseteq p^{2}(S, C)$, then $p^{3}(S, C) \subseteq p(S, C)$.

A directed, interpolation group $G$ is said to be a weak $C$-polar group if and only if $p^{3}(S, C)=p(S, C)$ for all subsets $S$ of $G$. The above lemma shows that every strong $C$-polar group is a weak $C$-polar group. The converse is false.

At this stage, it is probably helpful to illustrate the above results with an example. For this purpose, consider valuation groups.

Let $A$ be a p.o. set and $R_{\alpha} \neq\{0\}$ be a trivially ordered subgroup of the reals or a subgroup of $R$, and let $V=V\left(A, R_{\alpha}\right)$. If $V$ is an interpolation group (i.e., satisfies Teller's condition-see [17]), then if $v \in V^{+}, p(v)=\left\langle\left\{u \in V^{+}:\right.\right.$if $\alpha \in M(v)$ and $\beta \in M(u)$, then there does not exist $\gamma \in A$ such that $\gamma \leqq \alpha, \beta\}\rangle$. Hence all the polars of elements of $V$ can be found.

Now consider $A=Z^{+} \cup Z^{-} \cup\{0\}$ partially ordered as follows: let $\alpha, \beta \in A$; then $\alpha<\beta$ iff either

(i) $\alpha, \beta \in Z^{-}$and $\alpha<\beta$ in the usual ordering on $Z^{-}$, or

(ii) $\alpha=0$ and $\beta \in Z^{-}$, or

(iii) $\alpha \in Z^{+}, \beta \in Z^{-}$and $\alpha \geqq-\beta$ in the usual ordering on $Z^{+}$. 


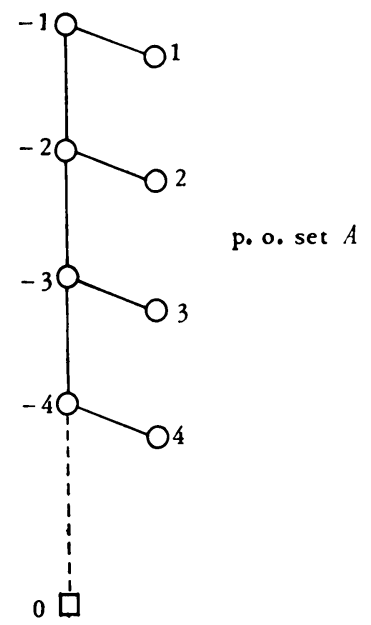

Let $R_{\alpha}=R$ if $\alpha \in A \mid\{0\}$ and let $R_{0}$ be the trivially ordered reals. $V=V\left(A, R_{\alpha}\right)$ is a directed, interpolation group by [17]. Let $v \in V\left(A, R_{\alpha}\right)$ be defined by

$$
\begin{aligned}
v_{\alpha}=0 & \text { if } \alpha \neq 0 \\
=1 & \text { if } \alpha=0
\end{aligned}
$$

Then $v \| 0$ and $u \geqq v, 0,-v$ iff there exists $\alpha \in M(u)$ such that $\alpha \in Z^{-}$. It is immediate that $p(v)=\left\{x \in V: x_{\alpha}=0\right.$ if $\alpha \in Z^{-} \cup\{0\}$ and there exists $\beta \in Z^{+}$such that $x_{\alpha}=0$ for all $\alpha \geqq \beta$ in the natural ordering of $\left.Z^{+}\right\}, p^{2}(v) \subseteq \mu\left(\left\{x \in V: x_{\alpha}=0\right.\right.$ if $\left.\left.\alpha=Z^{+} \cup Z^{-}\right\}\right)$ $=\{0\}$ (this can be seen by letting $n \in Z^{+}$and $x^{(n)} \in V^{+}$be defined by $x_{\alpha}^{(n)}=1$ if $\alpha=1, \ldots, n$ and $x_{\alpha}^{(n)}=0$ for all other $\alpha \in A$. $x^{(n)} \in p(v)^{+}$for every $n \in Z^{+}$and $p\left(x^{(n)}\right)=\left\langle\left\{y \in V^{+}: y_{\alpha}=0\right.\right.$ for all $\alpha \in Z^{+} \cup Z^{-}$such that $|\alpha| \leqq n$ in the natural ordering on $\left.\left.Z^{+}\right\}\right\rangle ; p^{2}(v) \subseteq \bigcap\left\{p\left(x^{(n)}\right): n \in Z^{+}\right\}=\mu\left(\left\{x \in V: x_{\alpha}=0\right.\right.$ if $\left.\left.\left.\alpha \neq 0\right\}\right)=\{0\}\right)$. Hence $p^{2}(v)=\{0\}$ and $v \notin p^{2}(v)$. Furthermore, $p(v) \subset G=p(0)=p^{3}(v)$. Thus $V$ is neither a weak $\{0\}$-polar nor a strong $\{0\}$-polar group. This is unlike the $l$-group result.

Let $A$ be defined as above and let $B=A \cup\{\overline{0}\}$. B has the same partial order as $A$ except that $\overline{0}<\alpha$ for all $\alpha \in Z^{-}$. Let $R_{\beta}=R$ if $\beta \in B \mid\{0\}$ and let $R_{0}$ be the trivially ordered reals. Let $W=\Sigma\left(B, R_{\beta}\right)$. Then $W$ is a weak $\{0\}$-polar group but, as above, it is not a strong $\{0\}$-polar group.

LEMMA 11. $p^{2}(S \cap T, C) \subseteq p^{2}(S, C) \cap p^{2}(T, C)$ for all subsets $S, T$ of $G$. If $S, T \in \mathscr{C}(G)$, then $p^{2}(S, C) \cap p^{2}(T, C)=p^{2}(S \cap T, C)$. Equality does not hold for arbitrary $S, T$ subsets of $G$, nor does $p^{2}\left(\bigcap\left\{S_{\alpha}: \alpha \in A\right\}, C\right)$ equal $\sqcap\left\{p^{2}\left(S_{\alpha}, C\right): \alpha \in A\right\}$ when $\left\{S_{\alpha}: \alpha \in A\right\} \subseteq \mathscr{C}(G)$, even if $G$ is an l-group.

Proof. The only difficulty is showing that if $S, T \in \mathscr{C}(G)$, then $p^{2}(S, C)^{+}$ $\cap p^{2}(T, C)^{+} \subseteq p^{2}(S \cap T, C)^{+}$. Let $0 \leqq x \in p^{2}(S, C) \cap p^{2}(T, C)$ and $0 \leqq y \in p(S \cap T, C)$. Let $s \in S^{+}$and $t \in T^{+}$. Suppose that $0 \leqq w \leqq x, y, s, t$. Then $w \in S \cap T$ and $0 \leqq w$ 
$\leqq w, y$. Thus $w \in C$, and so if $0 \leqq a \leqq x, y, s$, then $a \in p(t, C)^{+}$. This holds for all $t \in T^{+}$. Therefore, $a \in p(T, C)$ by Lemma 7(ii). Now $x \in p^{2}(T, C)$ and $0 \leqq a \leqq a, x$. Thus $a \in C$. Hence if $0 \leqq v \leqq x, y$ and $0 \leqq w \leqq v, s$, then $w \in C$, i.e. $v \in p(s, C)$ and this holds for all $s \in S^{+}$. Consequently, $v \in p(S, C)$. But $x \in p^{2}(S, C)$ and so $v \in C$. It now follows that $x \in p(y, C)$. Therefore $x \in p^{2}(S \cap T, C)$.

LEMMA 12. If $\left\{S_{\alpha}: \alpha \in A\right\}$ is a family of subsets of $G$, then $p\left(\bigcup\left\{S_{\alpha}: \alpha \in A\right\}, C\right)$ $=\mu\left(\bigcap\left\{p\left(S_{\alpha}, C\right): \alpha \in A\right\}\right)$. If for all $\alpha \in A, S_{\alpha} \in \mathscr{C}(G)$, then $p\left(\bigcup\left\{S_{\alpha}: \alpha \in A\right\}, C\right)$ $=p\left(\left\langle\bigcup\left\{S_{\alpha}: \alpha \in A\right\}\right\rangle, C\right)$.

LEMMA 13. If $\left\{S_{\alpha}: \alpha \in A\right\}$ is a family of subsets of $G$, then

$$
p\left(S_{\beta}, C\right) \subseteq p^{2}\left(\bigcup\left\{p\left(S_{\alpha}, C\right): \alpha \in A\right\}, C\right)=p^{2}\left(\left\langle\bigcup\left\{p\left(S_{\alpha}, C\right): \alpha \in A\right\}\right\rangle, C\right)
$$

for all $\beta \in A$. Moreover, $p^{2}\left(\bigcup\left\{p\left(S_{\alpha}, C\right): \alpha \in A\right\}, C\right)$ is the least $C$-polar of a weakly positive set which contains each $p\left(S_{\beta}, C\right)$. If $G$ is a weak $C$-polar group, then it is the least $C$-polar containing each $p\left(S_{\beta}, C\right)$.

That $p^{2}\left(\bigcup\left\{p\left(S_{\alpha}, C\right): \alpha \in A\right\}, C\right)$ is not the least $C$-polar containing each $p\left(S_{\beta}, C\right)$, in general, can be seen from the example on pp. 6 and 7 with $S_{\lambda}=\{v\}$ for all $\lambda \in \Lambda$.

Lemma 14. If $A, B \in \mathscr{C}(G)$, then $p^{2}(p(A, C) \cup p(B, C), C)=p(A \cap B, C)$.

Let $\mathscr{T}(G, C)=\{p(S, C): S$ is a subset of $G\}, \mathscr{W}(G, C)=\{p(S, C): S$ is a weakly positive subset of $G\}$ and $\mathscr{B}(G, C)=\{p(S, C): S \in \mathscr{C}(G)\}$.

LEMMA 15. $\mathscr{W}(G, C)=\mathscr{B}(G, C) . \mathscr{T}(G, C)=\mathscr{B}(G, C)$ if and only if $G$ is a weak C-polar group.

Notice that if $G$ is a directed, strong interpolation group (and so, in particular, if $G$ is an $l$-group), then $\mathscr{T}(G, C)=\mathscr{B}(G, C)$ for all $C \in \mathscr{C}(G)$. If $C=G, \mathscr{T}(G, C)$ $=\mathscr{B}(G, C)=\{G\}$. For the rest of this section, suppose $C \neq G$.

The machinery has now been established to prove the main theorem of this section concerning the structures of $\mathscr{B}(G, C)$ and $\mathscr{T}(G, C)$.

THEOREM 16. (i) $\mathscr{B}(G, C)$ is a complete Boolean algebra under inclusion, which is a complete lower subsemilattice of $\mathscr{C}(G)$, but not necessarily a sublattice of $\mathscr{C}(G)$, even if $G$ is an l-group. Moreover,

$$
\sqcup\left\{p\left(C_{\alpha}, C\right): \alpha \in A\right\} p(B, \sqcap C)=\bigsqcup\left\{p\left(C_{\alpha}, C\right) \sqcap p(B, C): \alpha \in A\right\}
$$

where $B, C_{\alpha} \in \mathscr{C}(G)(\alpha \in A)$.

(ii) $\mathscr{T}(G, C)$ is a complete lattice which is a complete lower subsemilattice of $\mathscr{C}(G)$ but not necessarily a sublattice of $\mathscr{C}(G)$ even if $G$ is an l-group. $\mathscr{T}(G, C)$ is not complemented, in general.

(iii) $\mathscr{B}(G, C)$ is a complete lower subsemilattice of $\mathscr{T}(G, C)$, but may not be a complete sublattice of $\mathscr{T}(G, C)$. 
Suppose that $\mathscr{L}, \mathscr{L}_{1}$ and $\mathscr{L}_{2}$ are lattices. Let $\pi_{1}, \pi_{2}$ be lattice homomorphisms of $\mathscr{L}$ into $\mathscr{L}_{1}, \mathscr{L}_{2}$ respectively. $\pi_{1} \geqq \pi_{2}$ iff (for all $L, M \in \mathscr{L}$, if $L \pi_{2}=M \pi_{2}$, then $L \pi_{1}=$ $\left.M \pi_{1}\right)$. If $\mathscr{L}_{1}$ has a least element, say 0 , then $\operatorname{ker}\left(\pi_{1}\right)=\left\{L \in \mathscr{L}: L \pi_{1}=0\right\}$.

TheOREM 17. Let $\pi_{c}: \mathscr{C}(G) \rightarrow \mathscr{B}(G, C)$ be defined by: $B \pi_{c}=p^{2}(B, C), B \in \mathscr{C}(G)$. $\pi_{c}$ is a lattice homomorphism of $\mathscr{C}(G)$ onto $\mathscr{B}(G, C)$ and $C$ is the largest element of $\operatorname{ker}\left(\pi_{c}\right)=\left\{B \in \mathscr{C}(G): B \pi_{c}=C\right\}$. Actually, if $\varphi$ is any lattice homomorphism of $\mathscr{C}(G)$ into a lattice with a 0 such that $C$ is the largest element of $\operatorname{ker}(\varphi)$, then $\varphi \leqq \pi_{C}$. This is a characterization of $\pi_{c}$.

Proof. We prove the last part of the theorem. Suppose $\mathscr{X}$ is a lattice with a 0 and that $\varphi: \mathscr{C}(G) \rightarrow \mathscr{X}$ is a lattice homomorphism with $C$ the largest element of $\operatorname{ker}(\varphi)$. Let $B \in \mathscr{C}(G)$ and define $\Delta(B)=\left\{M \in \mathscr{C}(G): M \varphi \Lambda_{\mathscr{X}} B \varphi=C \varphi\right\}$. If $M \in \Delta(B)$, then $M \cap B \subseteq C$, by hypothesis. Hence $M \subseteq p(B, C \cap B)=p(B, C)$ by Lemmas 9(iv) and 7(iv). Moreover, $(p(B, C) \cap B) \varphi=(p(B, C \cap B) \cap B) \varphi=(B \cap C) \varphi=C \varphi$. Thus $p(B, C) \in \Delta(B)$, and so is the largest element of $\Delta(B)$. Consequently, if $M \varphi=N \varphi, p(M, C)=p(N, C)$; therefore, $M \pi_{C}=N \pi_{C}$.

It should be observed that $\pi_{C}$ is a complete upper semilattice homomorphism. Another map that might be considered is $\varphi: \mathscr{B}(G, C) \rightarrow \mathscr{B}(G, C)$ defined by $p(B, C) \varphi=p^{2}(B, C), B \in \mathscr{C}(G)$.

THEOREM 18. If $\varphi: \mathscr{B}(G, C) \rightarrow \mathscr{B}(G, C)$ is defined by $p(B, C) \varphi=p^{2}(B, C)$, $B \in \mathscr{C}(G)$, then $\varphi$ is a complete lattice anti-isomorphism of $\mathscr{B}(G, C)$ onto $\mathscr{B}(G, C)$.

$B \in \mathscr{C}(G)$ is called a cardinal summand of $G$ if and only if there exists $A \in \mathscr{C}(G)$ such that $G=A \boxplus B$ and a $C$-cardinal summand of $G$ if and only if there exists $A \in \mathscr{C}(G)$ such that $A \cap B=C$ and $A \sqcup B=G$. Following Byrd [3], it will be shown that every $C$-summand (i.e. $C$-cardinal summand) is a $C$-polar and hence every cardinal summand is a polar. This is done in the following lemma.

LemmA 19. (i) If $G=A \boxplus_{C} B$ and $C \subseteq M \in \mathscr{C}(G)$, then $M=(M \cap A) \boxplus_{C}(M \cap B)$.

(ii) If $G=A \boxplus_{C} B$, then $A=p(B, C)$ and $B=p(A, C)$.

Proof. (ii) Suppose $G=A \boxplus_{C} B$. By Lemma 9(iv), $A \subseteq p(B, C)$. Let $x \in p(B, C)^{+}$. There exist $a_{1}, \ldots, a_{n} \in A^{+}$and $b_{1}, \ldots, b_{n} \in B^{+}$such that $x=a_{1}+b_{1}+\cdots+a_{n}+b_{n}$. Now $0 \leqq b_{i} \leqq b_{i}, x$ for $1 \leqq i \leqq n$. Hence $b_{i} \in C, 1 \leqq i \leqq n$. Thus $x \in\langle A \cup C\rangle^{+}=A^{+}$ since $C \subseteq A$. Consequently, $p(B, C) \subseteq A$ and the proof is complete.

Let $\mathscr{M}(G, C)=\{B \in \mathscr{C}(G): B$ is a $C$-summand of $G\}$. If $B \in \mathscr{M}(G, C)$, then $G=A \boxplus_{C} B$ for some $A \in \mathscr{C}(G)$ and $B=p(A, C)=p^{2}(B, C)$. However, $B \in \mathscr{C}(G)$ and $B=p^{2}(B, C)$ do not imply $B \in \mathscr{M}(G, C)$. This can be seen by taking $G$ to be the $l$-group of continuous real-valued functions and $B=\{f \in G: f(x)=0$ for all $x \geqq 0\}$. $B=p^{2}(B)$ but $B \sqcup p(B)=\{f \in G: f(0)=0\} \neq G$. Hence $B \notin \mathscr{M}(G, C)$.

TheOREM 20. $\mathscr{M}(G, C)$ is a distributive sublattice of both $\mathscr{B}(G, C)$ and $\mathscr{C}(G)$. Indeed, $\mathscr{M}(G, C)$ is a Boolean subalgebra of $\mathscr{B}(G, C)$ but not, in general, a complete Boolean subalgebra of $\mathscr{B}(G, C)$ even when $G$ is an l-group. 
4. Prime subgroups and $C$-polars. Instead of examining

$$
\mathscr{B}(G, C)=\{p(B, C): B \in \mathscr{C}(G)\}
$$

where $C$ is fixed, we now consider $\mathscr{P}(G, B)=\{p(B, C): C \in \mathscr{C}(G)\}$ for a fixed $B \in \mathscr{C}(G)$. By Lemma 7(iv),

$$
\mathscr{P}(G, B)=\{p(B, C): C \in \mathscr{C}(G) \text { and } C \subseteq B\}=\{p(B, C): C \in \mathscr{C}(B)\} .
$$

Observe that $\mathscr{P}(G, G)=\mathscr{C}(G)$.

A certain type of convex $d$-subgroup will play an important role in the following. $C \in \mathscr{C}(G)$ is said to be a prime subgroup of $G$ if and only if it is finitely meet irreducible in $\mathscr{C}(G)$, i.e. if $C_{1}, C_{2} \in \mathscr{C}(G)$ and $C=C_{1} \cap C_{2}$, then $C=C_{1}$ or $C=C_{2}$.

LEMMA 21. (i) $C$ is a prime subgroup of $G$ iff $C \in \mathscr{C}(G)$ and whenever $A, B \in \mathscr{C}(G)$ are such that $A \cap B \subseteq C$, then $A \subseteq C$ or $B \subseteq C$.

(ii) If $C$ is a prime subgroup of $G, a, b \in G^{+}$and $a \wedge b$ exists in $G$, then if $a \wedge b \in C$, either $a \in C$ or $b \in C$.

(iii) If $G$ is an l-group and $C \in \mathscr{C}(G)$, then the converse of (ii) is true.

Proof. (i) Suppose $C$ is a prime subgroup of $G, A, B \in \mathscr{C}(G)$ and $A \cap B \subseteq C$. Assume by way of contradiction, that $A, B \nsubseteq C$. Then there exist $a \in A^{+} \mid C$ and $b \in B^{+} \mid C . \quad a \in p^{2}(a, C) \in \mathscr{C}(G), \quad b \in p(a, C) \in \mathscr{C}(G)$ and $p(a, C) \cap p^{2}(a, C)=C$. Hence $p(a, C)=C$ or $p^{2}(a, C)=C$, since $C$ is prime. Thus $b \in C$ or $a \in C$, a contradiction. The converse is a fortiori.

(ii) Follows similarly.

The following lemma is easy to check:

LEMMA 22. If $G$ is a directed, interpolation group and $C \in \mathscr{C}(G)$, let $R(C)$ $=\{C+g: g \in G\}$, the right cosets of $C$ in $G$. Then $R(C)$ is a directed set satisfying the interpolation property under the ordering: $C+g \leqq C+h$ iff there exists $c \in C$ such that $c+g \leqq h$. Furthermore, if $g \vee h(g \wedge h)$ exists in $G$, then $C+x+g \vee C+x+h$ $(C+x+g \wedge C+x+h)$ exists in $R(C)$ and is equal to $C+x+(g \vee h)(C+x+(g \wedge h))$ and $C+g+x \vee C+h+x(C+g+x \wedge C+h+x)$ exists in $R(C)$ and is equal to $C+(g \vee h)+x(C+(g \wedge h)+x)$ for all $x \in G$.

If $G$ is an $l$-group and $C \in \mathscr{C}(G)$, then $\{B \in \mathscr{C}(G): C \subseteq B\}$ is totally ordered by inclusion if and only if $C$ is a prime subgroup of $G$. That this is not the case for directed, interpolation groups was shown in the example following Theorem B of [11]. Howevèr,

THEOREM 23. Let $G$ be a directed, interpolation group and $C \in \mathscr{C}(G)$. (1) $\rightarrow$ (2) $\leftrightarrow$ (3) but (2) $\rightarrow$ (1), in general, where (1), (2) and (3) are given by

(1) $\{B \in \mathscr{C}(G): C \subseteq B\}$ is an antilattice under inclusion.

(2) $C$ is a prime subgroup of $G$.

(3) $R(C)$ is an antilattice set under the partial order of the previous lemma. 
Proof. Since $\{B \in \mathscr{C}(G): C \subseteq B\}$ is a lattice under inclusion, the example cited above shows $(2) \rightarrow(1)$. That $(1) \rightarrow(2)$ is obvious.

(2) $\rightarrow$ (3). Suppose $C+g \wedge C+h$ exists and $=C+k$. Then $C+g-k \wedge C+h-k$ exists and $=C$. Hence, without loss of generality, $g, h \geqq 0$ and $k=0$. Since $C$ is prime, $p(g, C)=C$ or $p^{2}(g, C)=C$. It is easy to check that $h \in p(g, C)$ and, by the fact that $g \geqq 0, g \in p^{2}(g, C)$. Thus $g \in C$ or $h \in C$; so $C+g=C$ or $C+h=C$. A similar argument holds if $C+g \vee C+h$ exists.

(3) $\rightarrow$ (2). Similar to the proof of Lemma 21(i).

Using Lemma 22 , if $G$ is an $l$-group and $C$ is prime, then $R(C)$ is totally ordered. It is easy to see that $(2) \rightarrow(1)$ if $G$ is an $l$-group and so all three conditions are equivalent in this case.

TheOREM 24. Let $B \in \mathscr{C}(G)$. Then $\mathscr{P}(G, B)$ is a complete distributive lattice under inclusion, but it is not a sublattice of $\mathscr{C}(G)$ even if $G$ is an l-group. If $\varphi_{B}: \mathscr{C}(B)$ $\rightarrow \mathscr{P}(G, B)$ is defined by $C \varphi_{B}=p(B, C)$ where $C \in \mathscr{C}(B)$, then $\varphi_{B}$ is a complete lattice isomorphism of $\mathscr{C}(B)$ onto $\mathscr{P}(G, B)$. If $C$ is a prime subgroup of $G$, and $B \nsubseteq C$, then $C=(B \cap C) \varphi_{B}$. Moreover, $C$ is a prime subgroup of $B$ if and only if $p(B, C)$ is a prime subgroup of $G$ and $C$ is a value in $B$ if and only if $p(B, C)$ is a value in $G$ of some element of $B$. In particular, if $B=G(g)=\{x \in G: m g \leqq x \leqq n g$ for some $m, n \in Z\}$ and $g \in G^{+}$, then $C$ is a maximal convex $d$-subgroup of $B$ if and only if $p(B, C)$ is a value of $g$ in $G$.

Proof. By the lemmas of the previous section, it is easy to show that $\varphi_{B}$ is a complete lattice isomorphism of $\mathscr{C}(B)$ onto $\mathscr{P}(G, B)$. If $C$ is a prime subgroup of $G$ and $B \nsubseteq C$, then there exists $b \in B^{+} \mid C . C=p(B, C) \cap p^{2}(B, C)$ and $b \in p^{2}(B, C)$. Hence $C=p(B, C)=p(B, B \cap C)=(B \cap C) \varphi_{B}$. If $C$ is a prime subgroup of $B$ and $K, L \in \mathscr{C}(G)$ are such that $K \cap L=p(B, C)$, then $B \cap K \cap L=C$. Therefore, $B \cap K=C$ or $B \cap L=C$. Hence $K \subseteq p(B, C)$ or $L \subseteq p(B, C)$ (by Lemma 9(iv)) and so $p(B, C)$ is a prime subgroup of $G$. The converse is proved similarly. If $C$ is a value in $B$, say of $b \in B$, then it is easy to prove $p(B, C) \in \mathscr{M}_{G}(b)$. If $p(B, C)$ is a value in $G$ of $b \in B$, then $b \notin p(B, C) \cap B=C$. If $C \subset M \in \mathscr{C}(B)$, then $p(B, C) \subset p(B, M)$ so $b \in p(B, M) \cap B=M$. Thus $C$ is a value in $B$ of $b$. The example is given by an l-group.

The next theorem develops the relationship between prime and polar.

THEOREM 25. If $C, B \in \mathscr{C}(G)$ and $C \subset B$, then the following are equivalent:

(i) $C$ is a prime subgroup of $p^{2}(B, C)$.

(ii) $C$ is a prime subgroup of $B$.

(iii) $p(B, C)$ is a prime subgroup of $G$.

(iv) $p(B, C)=p(b, C)$ for all $b \in B^{+} \mid C$.

(v) $p(B, C)$ is a maximal $C$-polar of a convex d-subgroup of $G$.

(vi) $p^{2}(B, C)$ is a minimal $C$-polar of a convex $d$-subgroup of $G$.

(vii) $p^{2}(B, C)$ is a maximal convex $d$-subgroup with respect to the property of having $C$ a prime subgroup. 
Proof. (vii) $\rightarrow$ (i). A fortiori.

(i) $\rightarrow$ (ii). $B \in \mathscr{C}(G)$ and so $B \subseteq p^{2}(B, C)$. The result now follows at once.

(ii) $\leftrightarrow$ (iii). By Theorem 24 .

(ii) $\rightarrow$ (iv). For all $b \in B^{+}, p(B, C) \subseteq p(b, C)$. If there exists $b \in B^{+} \mid C$ such that $p(B, C) \neq p(b, C)$, then choose $x \in p(b, C)^{+} \backslash p(B, C)$. Now

$$
p(B, C)=\mu\left(\bigcap\left\{p(a, C): a \in B^{+}\right\}\right) .
$$

Hence there exists $a \in B^{+}$such that $x \notin p(a, C)$. Consequently, there exists $y \in G \backslash C$ such that $0 \leqq y \leqq a, x . B$ and $p(b, C)$ are convex. Thus $y \in B \cap p(b, C)$. But $C$ is a prime subgroup of $B$ (by (ii)) and so $C=p(b, C) \cap B$ or $C=p^{2}(b, C) \cap B$. However, $y \in(p(b, C) \cap B) \mid C$ and $b \in\left(p^{2}(b, C) \cap B\right) \mid C$, a contradiction. It now follows that $p(B, C)=p(b, C)$ for all $b \in B^{+} \backslash C$.

(iv) $\rightarrow$ (v). Suppose $C \subseteq D \in \mathscr{C}(G)$ and $p(B, C) \subseteq p(D, C) \subset G$. Now $D \ddagger p(B, C)$ (otherwise $D=D \cap p(B, C) \subseteq D \cap p(D, C)=C$ and so $p(D, C)=G$, a contradiction). Hence there exists $d \in D^{+} \backslash p(B, C)$. By Lemma 7(ii), there exists $b \in B^{+}$ such that $d \notin p(b, C)$. Thus there exists $u \in G \backslash C$ such that $0 \leqq u \leqq b, d$. Consequently, $u \in(D \cap B)^{+} \mid C$. By (iv), $p(D, C)=p(u, C)=p(B, C)$.

(v) $\rightarrow$ (vi). Trivial.

(vi) $\rightarrow$ (vii). Suppose that $C$ is not a prime subgroup of $p^{2}(B, C)$. Then there exist $K, L \in \mathscr{C}\left(p^{2}(B, C)\right)$ such that $C \subset K, L$ and $C=K \cap L$. Hence there exist $k \in K^{+} \mid C$ and $f \in L^{+} \mid C$. Let $N=G(k)=\{x \in G: n k \leqq x \leqq m k$ for some $m, n \in Z\}$ $\in \mathscr{C}(G) . N \subseteq K$. Let $M=\langle N \cup C\rangle \subseteq K \subseteq p^{2}(B, C)$. Therefore, $C \subset p^{2}(M, C) \subseteq p^{2}(B, C)$ since $k \in p^{2}(M, C) \backslash C$. By (vi), $p^{2}(M, C)=p^{2}(B, C)$ and so $p(M, C)=p(B, C)$. Let $x \in M^{+}$. Then $x \in K^{+}$, and as $K \cap L=C, f \in p(x, C)^{+}$. Consequently, $f \in p(M, C)=p(B, C)$. It follows that $f \in p(B, C) \cap L \subseteq p(B, C) \cap p^{2}(B, C)=C$, a contradiction. Hence $C$ is a prime subgroup of $p^{2}(B, C)$ and so (i) holds. By what has already been shown, (iv) holds. It is now easy to prove that if $p^{2}(B, C)$ $\subseteq D \in \mathscr{C}(G)$ and $C$ is a prime subgroup of $D$, then $p(D, C)=p(B, C)$ and so $D \subseteq p^{2}(D, C)=p^{2}(B, C)$. Thus $p^{2}(B, C)=D$. Q.E.D.

CoRollary 25.1. If $C \subset B, C, B \in \mathscr{C}(G)$ and $C$ is a prime subgroup of $B$, then the following are equivalent:

(a) $C$ is a prime subgroup of $G$.

(b) $p^{2}(B, C)=G$.

(c) $p(B, C)=C$.

THEOREM 26. If $C \in \mathscr{C}(G)$ and $C \neq G$, then the following are equivalent:

(1) $C$ is a prime subgroup of $G$.

(2) $\mathscr{T}(G, C)=2$.

(3) $\mathscr{B}(G, C)=2$.

The proof is easy.

COROLLARY 26.1. $\{0\}$ is a prime subgroup of $G$ if and only if $G$ is an antilattice. 
Proof. $G \neq\{0\}$ is an antilattice if and only if $\mathscr{T}(G,\{0\})=2$.

This concludes the section. One remarkable fact about the last two sections is that there is so much similarity between the l-group case and the directed, interpolation group case, despite the fact that "prime" is much weaker in the latter and $|g|$ may not exist if $g \| 0$.

5. $C$-carriers and principal $C$-bipolars. If $G$ is an $l$-group and $C$ is a convex $l$-subgroup of $G$, then $C$-carriers are defined (by Byrd) as equivalence classes of positive elements of $G$ with respect to $C$-polars, i.e. $g^{\wedge(C)}$ (the $C$-carrier of $g$ ) $=\left\{h \in G^{+} ; p(h, C)=p(g, C)\right\}$ for $g \in G^{+}$. It is clear that this definition can be used when $G$ is a directed, interpolation group and $C \in \mathscr{C}(G)$. In [9], Fuchs defined the carrier of $g \in G^{+}$to be $\left\{h \in G^{+}: X_{h}=X_{g}\right\}$ (where $X_{g}=\left\{k \in G^{+}: g \wedge k=0\right\}$ ) for $G$ a directed, interpolation group $(p(g)$ was not defined in [9]). His definition agrees with the one given here if $C$ is set equal to $\{0\}$. It is easy to see that

LEMMA 27. $g^{\wedge(C)}$ is a convex subsemigroup of $G^{+}$for all $g \in G^{+}$. Moreover, if $h \in g^{\wedge(C)}$ and $x, y \in G^{+}$, then $x+h+y \in(x+g+y)^{\wedge(C)}$.

Now for fixed $C \in \mathscr{C}(G)$, let $\mathscr{K}(G, C)\left(=\left\{g^{\wedge(C)}: g \in G^{+}\right\}\right.$. Define a partial order $\mathscr{K}(G, C)$ as follows: Let $g, h \in G^{+}$. Then $g^{\wedge(C)} \leqq h^{\wedge(C)}$ iff $p(h, C) \subseteq p(g, C)$. The following theorem extends a result (for $C=\{0\}$ ) of Fuchs. The proof is essentially the same as that given in [9].

THeOREM 28. $\mathscr{K}(G, C)$ is a directed set with a least element. For all $g, h \in G^{+}$, $(g+h)^{\wedge(C)}$ is the least upper bound of $g^{\wedge(C)}$ and $h^{\wedge(C)}$ in $\mathscr{K}(G, C)$. Hence $\mathscr{K}(G, C)$ is an upper semilattice. Indeed, $\mathscr{K}(G, C)$ is distributive in the sense that if $g^{\wedge(C)}$ $\wedge h^{\wedge(C)}$ exists in $\mathscr{K}(G, C)$, then so does $\left(g^{\wedge(C)} \vee f^{\wedge(C)}\right) \wedge\left(h^{\wedge(C)} \vee f^{\wedge(C)}\right)$, and it is equal to $\left(g^{\wedge(C)} \wedge h^{\wedge(C)}\right) \vee f^{\wedge(C)}$, and if also $g^{\wedge(C)} \wedge k^{\wedge(C)}$ exists, then so does $g^{\wedge(C)}$ $\wedge\left(h^{\wedge(C)} \vee k^{\wedge(C)}\right)$ and it is equal to $\left(g^{\wedge(C)} \wedge h^{\wedge(C)}\right) \vee\left(g^{\wedge(C)} \vee k^{\wedge(C)}\right)$. If $\mathscr{K}(G, C)$ is finite, then it is a Boolean algebra.

Lemma 29. Let $\varphi: G^{+} \rightarrow \mathscr{K}(G, C)$ be defined by $g \varphi=g^{\wedge(C)}$, for $g \in G^{+}$. Then $\varphi$ is an order-preserving map of $G^{+}$onto $\mathscr{K}(G, C)$, with kernel, $C^{+}$, which preserves all finite suprema and infima which exist in $G$. In the case that $G$ is an l-group, $\mathscr{K}(G, C)$ is a lattice and $\varphi$ is the maximal lattice homomorphism having $C^{+}$as kernel.

Proof. The first part is routine. The second part is due to an extension of a result of R. S. Pierce (see [8]). Cf. Theorem 17.

A structure which is for all practical purposes the same as $\mathscr{K}(G, C)$ is the set of principal C-bipolars of positive elements of $G, \mathscr{D}(G, C)=\left\{p^{2}(g, C): g \in G^{+}\right\}$. The next theorem shows that the two structures are tied together.

TheOREM 30. Let $\varphi: \mathscr{K}(G, C) \rightarrow \mathscr{D}(G, C)$ be given by $g^{\wedge(C)} \varphi=p^{2}(g, C)$. Then $\varphi$ and $\varphi^{-1}$ are upper semilattice isomorphisms that preserve finite infima whenever they exist. Also, $p^{2}(g, C)^{+}=\left\{h \in G^{+}: h \leqq k\right.$ for some $\left.k \in g^{\wedge(C)}\right\}$, for $g \in G^{+}$. 
Proof. It is easy to check that $p^{2}(g+h, C)$ is the least upper bound of $p^{2}(g, C)$ and $p^{2}(h, C)$, whenever $g, h \in G^{+}$(since $p^{3}(g, C)=p(g, C)$ if $g \in G^{+}$and $p(g+h, C)$ $=p(g, C) \cap p(h, C))$. Let $g \in G^{+}$and $S=\left\{h \in G^{+}: h \leqq k\right.$ for some $\left.k \in g^{\wedge(C)}\right\}$. If $h \in S$, then $h \in p^{2}(g, C)^{+}$. Conversely, if $h \in p^{2}(g, C)^{+}$, then $h \leqq g+h \in g^{\wedge(C)}$. It follows that $h \in S$ and so $S=p^{2}(g, C)^{+}$.

If $g^{\wedge(C)}=h^{\wedge(C)}$, then $p^{2}(g, C)=p^{2}(h, C)$ and if $g^{\wedge(C)} \neq h^{\wedge(C)}$, then $p(g, C) \neq$ $p(h, C)$; as $p^{3}\left(g^{\prime}, C\right)=p\left(g^{\prime}, C\right)$ for all $g^{\prime} \in G^{+}, p^{2}(g, C) \neq p^{2}(h, C)$. Consequently, $\varphi$ is an upper semilattice isomorphism.

This theorem extends Byrd's corresponding theorem for $l$-groups.

The section closes with a result linking $C$-carriers to prime subgroups (cf. Theorem 25).

THEOREM 31. Let $g \in G^{+} \mid C$. Then the following are equivalent:

(1) $g^{\wedge(C)}$ is a minimal C-carrier.

(2) $p(g, C)$ is a prime subgroup of $G$.

(3) $p^{2}(g, C)$ is a maximal convex $d$-subgroup with respect to the property of having $C$ a prime subgroup.

Proof. $g \in p^{2}(g, C) \mid C$ and $p^{3}(g, C)=p(g, C)$ so (2) and (3) are equivalent by Theorem 25.

(2) $\rightarrow(1)$. If $g^{\wedge(C)}$ is not a minimal $C$-carrier, then there exists $h \in G^{+} \mid C$ such that $h^{\wedge(C)}<g^{\wedge(C)}$. Hence $p^{3}(g, C) \subset p^{3}(h, C) \subset G$ and $p^{3}(g, C)$ is a maximal $C$-polar of a convex $d$-subgroup of $G$ (by Theorem 25), a contradiction.

$(1) \rightarrow(3)$. By Theorem 25 , it is enough to show that $C$ is a prime subgroup of $p^{2}(g, C)$. By way of contradiction, assume that $g^{\wedge(C)}$ is a minimal $C$-carrier and $C$ is not a prime subgroup of $p^{2}(g, C)$. Then there exist $K, L \in \mathscr{C}\left(p^{2}(g, C)\right)$ such that $K \cap L=C$ and $C \subset K, L$. Choose $k \in K^{+} \mid C$ and $f \in L^{+} \mid C$. By Theorem 30, there exist $h, h^{\prime} \in g^{\wedge(C)}$ such that $f \leqq h$ and $k \leqq h^{\prime}$. Hence $f^{\wedge(C)}, k^{\wedge(C)} \leqq g^{\wedge(C)}$ and as $k^{\wedge(C)}, f^{\wedge(C)} \neq 0^{\wedge(C)}, k^{\wedge(C)}=g^{\wedge(C)}=f^{\wedge(C)}$. But $k \notin p(k, C)$ and $k \in p(f, C)$ so $k^{\wedge(C)} \neq$ $f^{\wedge(C)}$, a contradiction.

COROLlary 31.1. Let $0<g \in G$. The following are then equivalent:

(1) $g^{\wedge}$ is a minimal carrier, where $g^{\wedge}=g^{\wedge((0))}$ and a carrier is a \{0\}-carrier.

(2) $p(g)$ is a prime subgroup of $G$.

(3) $p^{2}(g)$ is a maximal antilattice subgroup of $G$.

6. Filters and prime subgroups. Several results about l-groups have been obtained using ultrafilters on the nonnegative elements of an l-group (e.g., [1], [14] and [16]). In [2], Byrd showed that prime filters correspond to prime subgroups and, in this correspondence, ultrafilters correspond to minimal prime subgroups. This is not the case for directed, interpolation groups because $R(C)$ is not necessarily totally ordered even if $C$ is prime (see Theorem 23). However, a correspondence can be established between prime filters and prime subgroups. As before, $\hat{v}$ will be a directed, interpolation group. 
Let $N=G^{-}$. A nonempty subset $F$ of $G \backslash N$ is said to be a filter (on $G \mid N$ ) if and only if

(i) whenever $x, y \in G$ are such that $x \leqq y$ and $x \in F$, then $y \in F$, and

(ii) whenever $x, y \in F$, there exists $z \in F$ such that $z \leqq x, y$.

Observe that if $G$ is an l-group, then (ii) can be replaced by: whenever $x, y \in F$, then $x \wedge y \in F$ (this is the usual definition of filter).

A maximal filter is called an ultrafilter. A filter $F$ is said to be prime if and only if whenever $x, y \in G$ and $x+y \in F$, then $x \in F$ or $y \in F$. A filter is said to be normal if and only if it is invariant. In [2], Byrd showed that if $G$ is an l-group, then every ultrafilter is prime. This is not true for directed, interpolation groups. Let $G=\boldsymbol{R} \oplus \rightarrow Z$ where $Z$ is totally unordered (i.e., $(r, n)>0$ iff $r>0$ ). Let

$$
F=\{(r, n): r>0\} \cup\{(0,2)\} .
$$

$F$ is an ultrafilter which is not prime since $(0,1)+(0,1)=(0,2) \in F$ but $(0,1) \notin F$. An alternative definition of prime might be: $\mathscr{U}(g, h) \subseteq F$ implies that $g \in F$ or $h \in F$. With this definition, the same difficulty remains.

Observe that if $G$ is a semi-isolated, directed, interpolation group and $F$ is a prime filter, then if $x, y \in F$, it follows that $x+y \in F(x=(x+y)-y$ and $-y \notin F)$.

Suppose $C \in \mathscr{C}(G)$. Then $C$ is called a strong prime subgroup of $G$ if and only if $\{C+g: g \in G\}=R(C)$ is totally ordered. It is easy to see that if $C$ is a strong prime subgroup, then $\{A \in \mathscr{C}(G): C \subseteq A\}$ is a chain, and so $C$ is a prime subgroup of $G$. In [11] following Theorem $B$, an example was given of a prime subgroup of a directed, interpolation group which is not a strong prime subgroup. The same example showed that there exists $B \in \mathscr{C}(G)$ such that $B$ contains a prime subgroup (namely $\{0\}$ ) but is not itself a prime subgroup. However, if $B \in \mathscr{C}(G)$ and $B$ contains a strong prime subgroup, then $B$ is itself a strong prime subgroup. If $G$ is a directed, strong interpolation group and $\mathscr{N}$ is a chain of strong prime subgroups, then $\cap \mathscr{N}$ is a strong prime subgroup. However, if $G$ is only a directed, interpolation group, then $\Pi \mathscr{N}$ need not be a strong prime subgroup. The example on p. 6 shows this.

LEMMA 32. Let $C$ be a prime subgroup of $G$. Then $F(C)=\{g \in G: C<C+g\}$ is a filter which is normal if $C \triangleleft G$. If $C$ is a strong prime subgroup of $G$, then $F(C)$ is a prime filter. If $C_{1}, C_{2}$ are distinct prime subgroups of $G$, then $F\left(C_{1}\right)$ and $F\left(C_{2}\right)$ are distinct and if $C_{2}$ is a strong prime subgroup of $G$ and $C_{2} \subseteq C_{1}$, then $F\left(C_{1}\right) \subseteq F\left(C_{2}\right)$.

Proof. Routine verification using the fact that $R(C)$ is an antilattice if $C$ is prime. Notice that if $C$ is not a strong prime subgroup, then $F(C)$ is not necessarily a prime filter; e.g., let $G=R \boxplus^{*} \boldsymbol{R}$ (i.e., $(a, b)>0$ iff $a>0$ and $\left.b>0\right)$ and $C=\{(0,0)\}$. $(-1,2),(2,-1) \notin F(C)$ but $(1,1) \in F(C)$. Moreover, if $C$ is a prime subgroup of $G,\{g \in G: C+g \ddagger C\}$ is not in general a filter (for, with the above example, $(1,0),(0,1) \in\{g \in G: C+g \ddagger C\}$ but if $(x, y) \leqq(1,0),(0,1)$, then $(x, y) \in N)$. 
LEMMA 33. Let $F$ be a prime filter and $C(F)=\mu\left(\left\{g \in G^{+}: g \notin F\right\}\right)$. Then $C(F)$ is a prime subgroup of $G$. If $F$ is normal, then $C(F) \triangleleft G$. If $F_{1} \subset F_{2}$, then $C\left(F_{2}\right)$ $\subset C\left(F_{1}\right)$. In general, $F$ is a normal, prime ultrafilter does not imply that $C(F)$ is a strong prime subgroup.

Proof. That $C(F) \in \mathscr{C}(G)$ is trivial as is the normality. If $A, B \in \mathscr{C}(G)$ and $C(F) \subset A, B$, choose $a \in A^{+} \mid C(F)$ and $b \in B^{+} \mid C(F) . a, b \in F$, so there exists $z \in F$ such that $z \leqq a, b$. Hence there exists $x \in G$ such that $0, z \leqq x \leqq a, b$, and it follows that $x \in F$. Therefore, $x \notin C(F)$ and so $C(F) \subset A \cap B$. The rest of the proof is easy, the example being provided by $G=R \oplus \rightarrow C_{2}$, and $F=G \mid N$.

LEMMA 34. (i) If $C$ is a strong prime subgroup of $G$, then $C(F(C))=C$.

(ii) If $C$ is a minimal strong prime subgroup of $G$, then $F(C)$ is a maximal prime filter with respect to giving rise to a strong prime subgroup.

(iii) If $F$ is a prime filter and $C(F)$ is a strong prime subgroup of $G$, then $F \subseteq F(C(F))$. If $F$ is also an ultrafilter, then $F=F(C(F))$.

(iv) If $F$ is a prime ultrafilter and $C(F)$ is a strong prime subgroup of $G$, then $C(F)$ is a minimal strong prime subgroup of $G$.

The proof is routine.

If $F$ is a prime filter and $C(F)$ is a strong prime subgroup of $G$, then $F$ is said to be a strong prime filter. Strong prime filters which are ultrafilters will be called strong ultrafilters. Let $\mathfrak{A}(G)$ be the collection of all prime subgroups of $G$ and $\mathfrak{B}(G)$ the collection of all strong prime subgroups of $G$. Let $\mathfrak{E}(G)$ be the collection of all filters, $\mathfrak{D}(G)$ the collection of all prime filters and $\mathfrak{S}(G)$ the collection of all strong prime filters. Partially order these five sets by inclusion. Then

THEOREM 35. Let $\varphi: \mathfrak{A}(G) \rightarrow \mathfrak{C}(G)$ be given by $C \varphi=F(C)$, where $C \in \mathfrak{A}(G)$. Then $\varphi$ is $a(1: 1)$ map and if $\pi=\varphi \mid \mathfrak{B}(G)$, then $\pi$ is an order-inverting isomorphism of $\mathfrak{B}(G)$ onto $\mathbb{E}(G)$. If $\alpha: \mathfrak{D}(G) \rightarrow \mathfrak{A}(G)$ is given by $F \alpha=C(F)$, where $F \in \mathfrak{D}(G)$, then $\alpha$ is $a(1: 1)$ order-inverting map and $\alpha \mid \mathbb{E}(G)=\pi^{-1}$. If $F$ is a strong ultrafilter, then $F \alpha$ is a minimal strong prime subgroup of $G$. If $C$ is a minimal strong prime subgroup of $G$, then $C \varphi$ is a maximal element of $\mathfrak{E}(G)$ (but not necessarily a strong ultrafilter).

This theorem gives the correspondence between prime filters and prime subgroups for directed, interpolation groups.

7. A generalization of Lorenzen's theorem to directed, interpolation groups. An l-group $G$ is said to be representable if and only if it is a subcartesian product of o-groups, with the cardinal ordering (that is, there exists $\left\{H_{\alpha}: \alpha \in A\right\}$, a family of o-groups such that $G$ is an $l$-subgroup of $\Pi\left\{H_{\alpha}: \alpha \in A\right\}$, and the projection of $G$ into $H_{\alpha}$ is onto for all $\alpha \in A$ ). Lorenzen's theorem states that $G$ is a representable l-group if and only if the polars of elements of $G^{+}$are o-ideals of $G$. This is equivalent to the carriers being invariant (see [15]). 
Since every antilattice which is an l-group is an o-group, the concept of "representable" can be "extended" to directed, interpolation groups. A directed, interpolation group $G$ will be called a subcartesian product of $\left\{H_{\alpha}: \alpha \in A\right\}$, a family of directed, interpolation groups, iff $G$ is a subgroup of $\prod\left\{H_{\alpha}: \alpha \in A\right\}$, the projection, $\pi_{\alpha}$, of $G$ into $H_{\alpha}$ is onto and $\operatorname{ker}\left(\pi_{\alpha}\right) \in \mathcal{O}(G)$ for each $\alpha \in A . G$ is representable if and only if $G$ is " $l$ "-isomorphic (i.e., by a map which preserves all finite suprema and infima which exist in $G$ ) to a subcartesian product of some family of antilattices.

THEOREM 36. A directed, interpolation group $G$ is o-isomorphic to a subcartesian product of $\left\{H_{\alpha}: \alpha \in A\right\}$, a family of directed, interpolation groups, iff there exists $\left\{N_{\alpha}: \alpha \in A\right\}$, a family of o-ideals of $G$, such that $G / N_{\alpha} \cong_{0} H_{\alpha}$ for all $\alpha \in A$ and $\bigcap\left\{N_{\alpha}: \alpha \in A\right\}=\{0\}$. The o-isomorphism (of $G$ ) is an "l"-isomorphism iff for each $\alpha \in A$, the o-isomorphisms of $G / N_{\alpha}$ onto $H_{\alpha}$ are "l"-isomorphisms.

A directed, interpolation group $G$ is irreducible iff whenever $G$ is "l"-isomorphic to a subcartesian product of a family $\left\{K_{\beta}: \beta \in B\right\}$ of directed, interpolation groups, the projection $\pi_{\beta_{0}}$ is $(1: 1)$ for some $\beta_{0} \in B$, i.e. $G \cong{ }_{l} K_{\beta_{0}}$ for some $\beta_{0} \in B$.

In [12], P. Hall gave a definition of a monolithic group and defined the lith of a group. Analogously, a p.o. group $G$ is said to be monolithic if and only if $\Pi\{K \in \mathcal{O}(G): K \neq\{0\}\} \neq\{0\}$. If $G$ is a monolithic p.o. group, then lith $(G)$ $=\sqcap\{K \in \mathcal{O}(G): K \neq\{0\}\}$ is the unique minimal $(\neq\{0\}) o$-ideal of $G$. In [12], P. Hall gave an example of a group which has a unique minimal normal subgroup but is not monolithic. Adapting his example, let $G=V\left(A, R_{\alpha}\right)$ where $R_{\alpha}=R$ for all $\alpha \in A$ and $A=Z^{-} \cup\{0\}$ ordered by: $\alpha<\beta$ iff $\alpha, \beta \in Z^{-}$and $\alpha<\beta$ in the usual total ordering on $Z^{-} . G$ is an $l$-group (see [7]) and, for each $\alpha \in A, M_{\alpha}=\left\{g \in G: g_{\beta}=0\right.$ if $\beta \| \alpha$ or $\beta \geqq \alpha\} \in \mathcal{O}(G) \mid\{0\}$. $M_{0}$ is the unique minimal $o$-ideal of $G$ since $\cdots \subset M_{-3} \subset M_{-2}$ $\subset M_{-1}$ and, if $N_{\alpha}=\left\{g \in G: g_{\beta}=0\right.$ if $\left.\beta \geqq \alpha\right\} \supseteq M_{\alpha}$, then $\left\{N_{\alpha}: \alpha \in A\right\}=\mathscr{C}_{1}(G)$ (see the proof of Theorem D of [11]). However, $G$ is not monolithic as $\Pi\left\{M_{\alpha}: \alpha \in A\right\}$ $=\{0\}$. Indeed, $\cap\left\{M_{\alpha}: \alpha \in A\right\}=\{0\}$.

THEOREM 37. Let $G$ be a directed, interpolation group. Then $(1) \rightarrow(2),(1) \rightarrow(3)$, (3) $\leftrightarrow$ (4) but (3) $\rightarrow(2)$, (3) $\rightarrow(1)((3) \rightarrow$ (1) if $G$ is a strong interpolation, directed group) and (2) $\rightarrow(1),(2) \rightarrow(3)$ even if $G$ is an l-group, where

(1) $G$ is monolithic,

(2) $G$ has a unique minimal $(\neq\{0\})$ o-ideal,

(3) $\cap\{K \in \mathcal{O}(G): K \neq\{0\}\} \neq\{0\}$,

(4) $G$ is irreducible.

Proof. Trivial-the example on p. 6 guarantees that $(3) \nrightarrow(1)$.

The following lemma is of technical importance. Its proof is straightforward and therefore omitted.

LEMMA 38. If $N \in \mathcal{O}(G)$, then $\mathcal{O}(G / N)=\{M / N: M \in \mathcal{O}(G)$ and $N \subseteq M\}$. 
Let $\mathscr{X}$ be a property of directed, interpolation groups. A directed, interpolation group $G$ is said to be $\mathrm{R} \mathscr{X}$ (residually $\mathscr{X}$ ) if and only if $G$ is " $l$ "-isomorphic to a subcartesian product of directed, interpolation groups which have the property $\mathscr{X}$. This definition is based on P. Hall's definition for groups.

THEOREM 39. Every directed, interpolation group is residually irreducible. Every directed, strong interpolation group is residually monolithic.

Proof. Let $G$ be a directed, interpolation group and $g \in G \backslash\{0\}$. As in [11], there exists $K_{g} \in \mathcal{O}(G)$ maximal in $\mathcal{O}(G)$ with respect to not having $g$ as a member. $\bigcap\left\{K_{g}: g \in G \mid\{0\}\right\}=\{0\}$ and $G / K_{g}$ is irreducible by Lemma 38, for each $g \in G \mid\{0\}$. By Theorem 36, $G$ is residually irreducible. If $G$ is a directed, strong interpolation group, then $G / K_{g}$ is monolithic for each $g \in G \mid\{0\}$.

The example on p. 6 shows that there exist nonresidually monolithic directed, interpolation groups. This is unlike the group theory result (see [12]).

$G$ is a regular directed, interpolation group iff $p(g) \triangleleft G$ for all $g \in G^{+}$.

LEMMA 40. Let $G$ be a directed, interpolation group. The following are equivalent:

(1) $G$ is regular.

(2) $p(S) \triangleleft G$ for all $S \subseteq G$.

(3) If $-x+g+x \in p(g)$ for some $x \in G$, then $g=0$.

(4) The carriers of positive elements of $G$ are invariant.

The proof is omitted since it is quite easy.

The next lemma leads to a generalization of Lorenzen's Theorem.

LEMMA 41. Let $G$ be a directed, interpolation group. If $G$ is regular and irreducible, then $G$ is an antilattice. If $G$ is an antilattice, then $G$ is regular.

Proof. If $G$ is regular and irreducible and $b \in p(a)^{+}\left(a \in G^{+}\right)$, then, by Lemma 40, $p(a), p^{2}(a) \in \mathcal{O}(G)$. In addition, $p(a) \cap p^{2}(a)=\{0\}$ by Lemma 9(i). By Theorem 37, (4) $\rightarrow(3), p(a)=\{0\}$ or $p^{2}(a)=\{0\}$. But $b \in p(a)$ and $a \in p^{2}(a)$ by Lemma 8(i). Hence $b=0$ or $a=0$; so $G$ is an antilattice.

If $G$ is an antilattice, then $\mathscr{T}(G,\{0\})=2$, so $G$ is regular.

TheOrem 42 (Generalization of Lorenzen's TheOrem). If $G$ is a directed, interpolation group, then $G$ is regular if and only if $G$ is representable.

Proof. Suppose that $G$ is regular. By Theorem 39, every directed, interpolation group is " $l$ "-isomorphic to a subcartesian product of irreducible, directed, interpolation groups. Since $G$ is regular, each of the irreducible, directed, interpolation groups is regular, as can be seen by the construction used in the proof of Theorem 39. By Lemma $41, G$ is representable.

The converse is immediate from Lemma 41. 
The following corollary gives the result proved by L. Fuchs in [9].

COROllary 42.1. Every abelian, directed, interpolation group is representable.

It should be noted that the construction given in Theorem 39 (which is crucial to the proof given of Lorenzen's Theorem) when applied to an $l$-group, $G$, gives $G$ $l$-isomorphic to a subcartesian product of irreducible l-groups. Lemma 41 now gives; If $G$ is a regular, irreducible $l$-group, then $G$ is an $o$-group. As a result, Lorenzen's Theorem for $l$-groups is recaptured.

Notice that by Lorenzen's Theorem, Theorem 42 and the fact that if $G$ is an $l$-group then the $l$-group definition of polar is the same as that given in this paper, it follows that an l-group is representable if and only if it is representable in the sense given here.

8. $o$-simple directed, interpolation groups. In group theory, one problem which has attracted a lot of study is characterizing simple groups. In the theory of p.o. groups, two problems can be posed. The first is to characterize those directed, interpolation groups which have no nontrivial $o$-ideals, and the second (which is easier) is to characterize those directed, interpolation groups which have no nontrivial normal, convex subgroups.

The second is easy to answer, provided the groups are abelian and weakly semi-isolated. The answer is

If $G$ is an abelian, directed interpolation, weakly semi-isolated group having only the trivial convex subgroups, then $G$ is an o-group and so o-isomorphic to a subgroup of $\boldsymbol{R}$. Indeed, if $G$ is an abelian, weakly semi-isolated group without nontrivial convex subgroups, then $G$ is a trivially ordered group of prime order or a subgroup of $\boldsymbol{R}$.

(If $g \in G$ and $g \| 0$, then $\langle g\rangle$ is a convex normal subgroup of $G$ and $\langle g\rangle \neq\{0\}$. Hence $G$ is trivially ordered and clearly of prime order. If $G$ is not of this form, then $G$ is an $o$-group. The rest of the theorem follows from a result of Hölder whose proof is provided in [8].)

The first problem is more difficult, even when abelian, weakly semi-isolated, directed, interpolation groups are considered. A p.o. group $G$ is said to be o-simple if and only if $\mathcal{O}(G)=\{G,\{0\}\}$.

THEOREM 43. If $G$ is a directed, interpolation group which is o-simple, then $G$ is irreducible. If $G$ is a representable directed, interpolation group which is o-simple, then $G$ is an antilattice. In particular, any o-simple, abelian, directed, interpolation group is an antilattice.

Proof. The first part follows from Theorem 37, whereas the second part follows from this, together with Theorem 42 and Lemma 41.

The last statement in Theorem 43 is known for p.o. vector spaces and is due to L. Fuchs (see Riesz vector spaces and Riesz algebras, Queen's Papers in Pure and Appl. Math., no. 1). 
A p.o. group $G$ is said to be strong o-simple if and only if $\mathscr{C}(G)=\{G,\{0\}\}$. Hence: If $G$ is a strong o-simple, directed, interpolation group, then $G$ is an antilattice (since $\{0\}$ is prime). This gives an alternative proof to the last part of Theorem 43.

The $o$-simple, abelian antilattices can be very varied. They may or may not be torsion-free, even if weakly semi-isolated. For example, let $G=\boldsymbol{R} \oplus \rightarrow C_{\infty}$ and $H_{n}=\boldsymbol{R} \oplus \rightarrow C_{n}$ where $n \in Z^{+} . G$ is torsion-free but no $H_{n}$ is. However, $G$ and $H_{n}$ (for all $n \in Z^{+}$) are abelian, $o$-simple, weakly semi-isolated antilattices. Indeed, it is easy to see that

THEOREM 44. A directed, interpolation group is strong o-simple if and only if it is an antilattice which is generated by any positive interval in its open interval topology.

For the rest of this section, an attempt will be made to show that in some sense, all $o$-simple, weakly semi-isolated, abelian p.o. groups are of the form $S \oplus \rightarrow T$ where $S$ is a group of real valued functions and $T$ is a trivially ordered group.

Let $G$ be an arbitrary p.o. group $\neq\{0\}$. By Zorn's Lemma, there exist maximal trivially ordered (convex) subgroups of $G$, provided that $G$ is not trivially ordered. $g \in G \backslash\{0\}$ is said to be pseudo-positive if and only if $h<g$ for all $h \in G$ such that $h<0$ and $g^{\prime} \in G \backslash\{0\}$ is said to be a pseudo-identity if and only if $h<g^{\prime}<f$ for all $h, f \in G$ such that $h<0<f$. This definition is due to L. Fuchs and originated in [9].

THEOREM 45. Let $\left\{K_{\alpha}: \alpha \in A\right\}$ be the set of all maximal trivially ordered subgroups of a p.o. group $G$ which is not trivially ordered, and let $K$ be the set of all pseudoidentities together with 0 . Then $K$ is a normal trivially ordered subgroup of $G$ and $K \subseteq \bigcap\left\{K_{\alpha}: \alpha \in A\right\}$. If $G$ is weakly semi-isolated, then $K=\bigcap\left\{K_{\alpha}: \alpha \in A\right\}$.

Proof. Clearly, $K$ is a normal trivially ordered subgroup of $G$. If $K \nsubseteq K_{\beta}$ for some $\beta \in A$, choose $k \in K \backslash K_{\beta}$. $\left\langle k, K_{\beta}\right\rangle$ is a subgroup of $G$ and so $n k>g$ for some $g \in K_{\beta}$ and $n \in Z$. Since $n k \in K$, it follows that $g<0$. Therefore, $K_{\beta}$ is not trivially ordered, a contradiction. Consequently, $K \subseteq \bigcap\left\{K_{\alpha}: \alpha \in A\right\}$.

If $G$ is weakly semi-isolated and $g \in \bigcap\left\{K_{\alpha}: \alpha \in A\right\}$, then either $g=0$ (whence $g \in K$ ) or $g \neq 0$. If $g \neq 0$, let $h>0 . h+g \notin K_{\alpha}$ for all $\alpha \in A$ since each $K_{\alpha}$ is trivially ordered. If $h+g \leqq 0$, then $g \leqq 0$, which is impossible, and if $h+g \| 0$, then $\langle h+g\rangle$ is trivially ordered and so $h+g \in\langle h+g\rangle \subseteq K_{\beta}$ some $\beta \in A$-a contradiction. It follows that $h+g>0$ and so $g>k$ for all $k \in G$ such that $k<0$. Similarly, $-g>k$ for all $k \in G$ such that $k<0$. Hence $g \in K$.

A p.o. group $G$ is said to be dense iff $f, g \in G$ and $f<g$ imply there exists $h \in G$ such that $f<h<g$.

THEOREM 46. If $G$ is an interpolation group and $K=\{g \in G: g=0$ or $g$ is a pseudoidentity $\} \neq\{0\}$, then $G$ and $G / K$ are dense interpolation groups, $G / K$ has no pseudoidentities and no suprema or infima exist in $G$ or $G / K$ of unrelated elements. If $G$ is directed (semi-isolated), then so is $G / K$. 
THEOREM 47. If $G$ is an abelian, semi-isolated group, then $G$ can be o-embedded in its divisible closure, $\bar{G}$, where $\bar{G}^{+}=\left\{x \in \bar{G}: n x \in G^{+}\right.$for some $\left.n \in Z^{+}\right\}$. $\bar{G}$ is an abelian, semi-isolated group. If $G$ is a directed (interpolation), abelian, semi-isolated group, then so is $\bar{G}$.

THEOREM 48. If $G$ is an abelian, divisible, semi-isolated group without pseudoidentities, then $G$ can be o-embedded in a mild cartesian product of divisible o-groups (i.e., $h>0$ iff $h_{\alpha}>0$ for all $\alpha \in A$ ).

The proofs of these theorems, which are generalizations of those in [9], are the same as those given there; for this reason, they are omitted here. Theorem 48 is proved as follows: For each $g>0$, let $K_{g}$ be a trivially ordered subgroup of $G$ maximal with respect to not containing $g . G / K_{g}$ is a divisible $o$-group and the canonical map of $G$ into the mild cartesian product of $G / K_{\alpha}\left(\left\{K_{\alpha}: \alpha \in A\right\}\right.$ is the set of all trivially ordered subgroups of $G$ maximal with respect to missing some $\left.g \in G^{+} \mid\{0\}\right)$ is an $o$-embedding.

If $G$ is an abelian, weakly semi-isolated group and $K$ is the subgroup of 0 together with the pseudo-identities of $G$, then $T(G) \subseteq K$ as was shown in [10], where $T(G)$ $=\{g \in G: g=0$ or $g$ is periodic $\} . G / K$ is semi-isolated under the induced order and so can be $o$-embedded in its divisible closure by Theorem 47. Let $H$ be the divisible closure of $G / K$. Then $H$ can be $o$-embedded in a mild cartesian product of divisible $o$-groups which are dense by Theorem 48 .

LEMMA 49. (1) If $G$ is an abelian, weakly semi-isolated group which is o-simple, then so is $G / K$.

(2) $G / K$ is o-simple if and only if $H$ is o-simple. In this case, each factor of the mild cartesian product given by Theorem 48 is o-simple and so is o-isomorphic to a subgroup of $\boldsymbol{R}$.

The proof is trivial-the fact that each factor is $o$-simple stems from the construction given in the proof of Theorem 48.

If $G$ is an abelian, weakly semi-isolated group without pseudo-identities, then $G$ is semi-isolated as it has no torsion elements $(T(G) \subseteq K)$. Hence

THEOREM 50. If $G$ is an o-simple, abelian, weakly semi-isolated group without pseudo-identities, it can be o-embedded in a mild cartesian product of divisible subgroups of $\boldsymbol{R}$.

If $G$ has pseudo-identities, then $G$ is a lexicographic sum of $G / K$ over $K$, where $K$ is the subgroup comprising the pseudo-identities of $G$ and 0 . Consequently, $G$ can be $o$-embedded in a lexicographic sum of a mild cartesian product of $o$ groups over a trivially ordered subgroup. Thus

THEOREM 51. If $G$ is an o-simple, abelian, weakly semi-isolated group with pseudo-identities, then $G$ can be o-embedded in a lexicographic sum of a mild cartesian product of divisible subgroups of $\boldsymbol{R}$ by a trivially ordered group. 
Notice, however, that this $o$-embedding into real valued functions on some trivially ordered set-or a lexicographic sum thereof-may not be an embedding into an $o$-simple p.o. group (if $\alpha$ is a limit ordinal, then $\Pi^{*}\left\{R_{\beta}: \beta<\alpha\right\}$ is not $o$-simple, where $R_{\beta}(\neq\{0\})$ is a divisible subgroup of $R$, for all $\beta<\alpha$. (Let

$$
L=\left\{f \in \prod^{*}\left\{R_{\beta}: \beta<\alpha: \lim _{\beta \rightarrow \alpha} f(\beta)=0\right\} \in \mathcal{O}\left(\prod^{*}\left\{R_{\beta}: \beta<\alpha\right\}\right)\right.
$$

and $L \neq\{0\}$ or $\Pi^{*}\left\{R_{\beta}: \beta<\alpha\right\}$.)). Thus the $o$-embedding leaves much to be desired.

9. An embedding theorem for directed, interpolation groups. In [13], Holland proved that every $l$-group can be $l$-embedded in the order-preserving permutations of a totally ordered set. In this section, his construction is imitated to prove that every directed, interpolation group can be "l"-embedded in the order-preserving permutations of an antilattice set. Holland's result is then recovered for $l$-groups. The key to the construction is examining the set of right cosets of a prime subgroup.

Let $G$ be a directed, interpolation group and $C \in \mathscr{C}(G) . A(C)$ is the group of all order-preserving permutations of the p.o. set $R(C)$ ordered by: $0 \leqq \pi \in A(C)$ iff $X \leqq X \pi$ (in $R(C)$ ) for all $X \in R(C)$. It is easy to verify that

LEMMA 52. Let $G$ be a directed, interpolation group and $C \in \mathscr{C}(G)$. Consider the map $\varphi: G \rightarrow A(C)$ defined by $(C+x) g \varphi=C+x+g$, for all $x, g \in G$. Then $\varphi$ is an "l"-homomorphism of $G$ onto $G \varphi$ and $\operatorname{ker}(\varphi) \subseteq C$. Moreover, $G \varphi$ is a directed subgroup of $A(C)$ that is transitive on $R(C)(H$ is transitive on a p.o. set $S$ iff for all $s, t \in S$ there exists $h \in H$ such that $s h=t)$.

Note that $\operatorname{ker}(\varphi)=\bigcap\{-x+C+x: x \in G\}$. If $C \in \mathcal{O}(G)$, then $C=\operatorname{ker}(\varphi)$ and $G \varphi \cong 0 G / C$ is a directed, interpolation group. However, if $G$ is the nonabelian $o$-simple $o$-group given in [5] and $H=G \oplus \rightarrow Z$ where $Z$ is trivially ordered, let $C^{\prime}$ be any convex subgroup of $G$ other than $G$ or $\{0\}$. Let $C=C^{\prime} \oplus \rightarrow Z$. Then $H$ is a directed, interpolation group and $C$ is a convex $d$-subgroup of $H$ which is prime. In this case, $\operatorname{ker}(\varphi)=\boldsymbol{Z}$ (trivially ordered) so $\operatorname{ker}(\varphi)$ is not directed. It is unknown as to whether $A(C)$ is a directed group or an interpolation group, in general. However

THEOREM 53. Let $G$ be a directed, interpolation group. Then $G$ is " $l$ "-isomorphic to a subgroup of a cartesian product of directed p.o. groups $\left\{B_{g}: g \in G\right.$ and $\left.g \$ 0\right\}$, such that each $B_{g}$ is a transitive subgroup of $A\left(C_{g}\right)$ for some prime subgroup $C_{g}$.

Proof. Let $g \in G$ and $g \neq 0$. Let $\mathscr{S}_{g}=\{B \in \mathscr{C}(G): B \cap \mathscr{U}(g)=\varnothing\}$. $\mathscr{S}_{g}$ satisfies the hypotheses of Zorn's Lemma, and so there exists $C_{g}$ which is maximal in $\mathscr{S}_{g}$. If $A, B \in \mathscr{C}(G), A \cap B=C_{g}$ and $A, B \neq C_{g}$, then there exist $a \in A^{+}$and $b \in B^{+}$such that $a, b \geqq g$ (since $A$ and $B$ are directed, $a$ and $b$ can be taken to be positive). Thus $a, b \geqq g, 0$ so there exists $k \in G$ such that $a, b \geqq k \geqq g, 0$. Now $k \in A \cap B=C_{g}$ and $k \in \mathscr{U}(g)$, a contradiction. Hence $C_{g}$ is prime, and by Theorem $23, R\left(C_{g}\right)$ is an antilattice set. Let $\varphi_{g}: G \rightarrow A\left(C_{g}\right)$ be given by Lemma 52 and let $B_{g}=G \varphi_{g} . B_{g}$ has the requisite properties by the previous lemma. Let $H=\prod\left\{B_{g}: g \in G\right.$ and $\left.g \$ 0\right\}$ 
with the usual cardinal ordering and let $\varphi: G \rightarrow H$ be given by $(g \varphi)_{k}=g \varphi_{k}$ for each $g \in G$ and each $k \in G$ such that $k \neq 0$. Clearly $\varphi$ is an "l"-homomorphism of $G$ onto $G \varphi$. It is $(1: 1)\left(g \notin C_{g}\right.$ for all $g \nsubseteq 0$, and so $\{0\}=\bigcap\left\{C_{g}: g \in G\right.$ and $\left.g \nsubseteq 0\right\}$. By Lemma $52, \operatorname{ker}\left(\varphi_{g}\right) \subseteq C_{g}$. But $\operatorname{ker}(\varphi)=\bigcap\left\{\operatorname{ker}\left(\varphi_{g}\right): g \in G\right.$ and $\left.g \neq 0\right\}$. Hence $\operatorname{ker}(\varphi)=\{0\}$.) and an $o$-epimorphism. (Assume $g \varphi \leqq f \varphi$. Then $f \nless g$. If $f \| g$, then $g-f \leqq 0$ and $C_{g-f}+g-f \leqq C_{g-f}$ since $g \varphi \leqq f \varphi$. Now there exists $c \in C_{g-f}$ such that $g-f \leqq c$; so $c \in C_{g-f} \cap \mathscr{U}(g-f)$, a contradiction. Thus $g \nmid f$; therefore $\left.g \leqq f\right)$ which completes the proof, as $\varphi$ is an "l"-embedding of $G$ into $H$.

THEOREM 54. If $G$ is a directed, interpolation group, then $G$ is "l"-isomorphic to a subgroup of the group of all order-preserving permutations of an antilattice set.

Proof. Totally order the nonnegative elements of $G$, say $\left\{g_{\alpha}: \alpha \in A\right\}$, where $A$ is a totally ordered set. Let $C_{g_{\alpha}}$ be defined as in the proof of Theorem 53 and $S_{\alpha}=R\left(C_{g_{\alpha}}\right)$. Without loss of generality, $S_{\alpha} \cap S_{\beta}=\varnothing$ for all $\alpha, \beta \in A$ such that $\alpha \neq \beta$. Let $S=\bigcup\left\{S_{\alpha}: \alpha \in A\right\}$ and define $s \leqq t$ iff $s \in S_{\alpha}$ and $t \in S_{\beta}$ and $\alpha<\beta$ or $\alpha=\beta$ and $s \leqq t$ in $S_{\alpha} . S$ is an antilattice set with respect to this partial ordering. Let $\varphi_{g_{\alpha}}$ be as in the proof of Theorem 53 and define $\varphi: G \rightarrow K$, where $K$ is the group of all orderpreserving permutations of $S$, by: let $s \in S$, so $s \in S_{\alpha}$ for a unique $\alpha \in A$. Let $s(g \varphi)=s\left(g \varphi_{g_{\alpha}}\right) \in S_{\alpha}$. Hence, if $g \in G$, then $g \varphi \mid S_{\beta}$ has range $S_{\beta}$ for all $\beta \in A$. $\varphi$ is an "l"-homomorphism of $G$ into $K$, and, by the previous observation, $\operatorname{ker}(\varphi)$ $=\bigcap\left\{\operatorname{ker}\left(\varphi_{g_{\alpha}}\right): \alpha \in A\right\}=\{0\}$, as in the proof of Theorem 53. As before, $\varphi$ is an $o$-homomorphism of $G$ onto $G \varphi$. $G \varphi$ is thus a directed, interpolation subgroup of $K$ and $\varphi$ is an "l"-embedding of $G$ into $K$. Q.E.D.

To conclude this section, Holland's original theorem is recovered.

Corollary 54.1 (Holland). If $G$ is an l-group, then $G$ is l-isomorphic to an l-subgroup of the l-group of all order-preserving permutations of a totally ordered set.

Proof. It was noted after Theorem 23 that $R(C)$ is a totally ordered set if $C$ is a prime subgroup of an $l$-group $G$. Consequently, each $S_{\alpha}$ is a totally ordered set and, therefore, so is $S$ (notation of the proof of Theorem 54). Since $\varphi$, given in the proof of Theorem 54, is an "l"-embedding of $G$ into $K$, it is an $l$-isomorphism between $G$ and $G \varphi$, an $l$-subgroup of the order-preserving permutations of the totally ordered set, $S$. The proof is completed by the observation that if $T$ is a totally ordered set, the order-preserving permutations of $T$ form an $l$-group with the ordering given on p. 22.

In [13], Holland went on to prove, with the aid of the Generalized Continuum Hypothesis, that every $l$-group can be $l$-embedded in a divisible $l$-group. E. C. Weinberg improved on the result by showing that one need not assume the Generalized Continuum Hypothesis to get the result (see [18]). Many other results concerning $l$-groups are obtained from Corollary 54.1 . These results are not readily accessible for directed, interpolation groups because of the more complicated structure of antilattice sets. In fact, the limitations in the study of directed, 
interpolation groups are owing to the lack of knowledge concerning antilattices. The last two sections illustrate this point very clearly.

10. Concluding remarks and open questions. Let $G$ be a directed, interpolation group and let Aut $(G)$ be the group of all $o$-automorphisms of $G$. $C \in \mathscr{C}(G)$ is said to be a shifting subgroup of $G$ if and only if $C \varphi=C$ or $C \varphi \cap C=\{0\}$ for all $\varphi \in$ Aut $(G)$ and a characteristic subgroup of $G$ if and only if $C \varphi=C$ for all $\varphi \in$ Aut $(G)$. Let $K(G)$ be the set of all characteristic subgroups of $G$ and $\mathscr{D}(G)$ the set of all shifting subgroups of $G$. $G$ is said to be $K$-simple (characteristically simple) iff $K(G)=\{G,\{0\}\}$.

In [4], Byrd, Conrad and Lloyd examined the characteristic and shifting subgroups of an l-group $G$. In $\$ 2,3$ and 4 of [4], results were obtained for a general $l$-group $G$. From the results on polars in this paper, almost all of the theorems of [4] from those sections hold with minor modifications for a directed, interpolation group. The proofs are the same as those given in [4].

Problems. (1) Is $\mathscr{B}(G, C)$ a sublattice of $\mathscr{T}(G, C)$ for every directed, interpolation group $G$ and $C \in \mathscr{C}(G)$ ?

(2) Give a complete characterization of the $o$-simple abelian antilattices.

(3) Find necessary and sufficient conditions on an antilattice set $S$ for the group of all order-preserving permutations of $S$ to be a directed, interpolation group.

(4) Can every semi-isolated, directed, interpolation group be "l"-embedded in a divisible, semi-isolated, directed, interpolation group?

(5) If $G$ has a finite number of polars, then characterize the directed, interpolation group $G$.

(6) Let $V=V\left(A, R_{\alpha}\right)$ where $A$ is an arbitrary p.o. set and $R_{\alpha}=R$ for all $\alpha \in A$. Give necessary and sufficient conditions for $V$ to be characteristically simple.

(7) Which abelian p.o. groups (not necessarily weakly semi-isolated) have no nontrivial convex subgroups?

With respect to Problem (5), it should be observed that if $G$ is a directed, interpolation group with precisely $n$ polars and $H$ is an arbitrary directed, interpolation group, then $H \oplus \rightarrow G$ is a directed, interpolation group with precisely $n$ polars. For this reason, it may be necessary to impose some extra conditions on $G$ to obtain any result.

With regard to Problem (6), it is easy to see that if $A$ has a maximal or a minimal element (i.e., there exists $\alpha \in A$ such that $\alpha \$ \beta$ for all $\beta \in A$ or $\alpha \geq \beta$ for all $\beta \in A$ ), then $V$ is characteristically simple if and only if $A$ is a finite trivially ordered set. In particular, this answers the problem in the case that $A$ is finite. In general, $A$ can be expressed as the disjoint union of sets $S_{\beta}(\neq \varnothing)$ which have the property that if $\gamma \in S_{\beta}, \alpha \in A$ and $\alpha \geqq \gamma$ or $\alpha \leqq \gamma$, then $\alpha \in S_{\beta}$. If $V$ is characteristically simple, then there can only be a finite number of $S_{\beta}$ 's and they are order-isomorphic. However, the $S_{\beta}$ 's may have infinite trivially ordered subsets; for any cardinal $m$, the set of maximal trivially ordered subsets of any $S_{\beta}$ is either empty or coinitial and cofinal in $S_{\beta}$. 
In Problem (7), if $G$ is a cyclic p.o. group, say $G=\langle g\rangle$, then $G$ has no nontrivial convex subgroups if and only if there exists $m \in Z^{+}$such that $n g \in G^{+}$for all $n \geqq m$ or $n g \in G^{+}$for all $n \leqq-m\left(n \in Z^{+} \cup Z^{-}\right)$.

Of course, in Problem (2), if the $o$-simple abelian antilattice is a $\varnothing$-group, then the group is an $o$-group and so $o$-isomorphic to a subgroup of $\boldsymbol{R}$.

\section{BIBLIOGRAPHY}

1. B. Banaschewski, On lattice-ordered groups, Fund. Math. 55 (1964), 113-122. MR 29 \#5930.

2. R. D. Byrd, Lattice-ordered groups, Ph.D. Dissertation, Tulane University, New Orleans, La., 1966.

3. —_, M-polars in lattice-ordered groups, Czechoslovak Math. J. 18 (93) (1968), 230-239. MR 37 \#2651.

4. R. D. Byrd, P. F. Conrad and J. T. Lloyd, Characteristic subgroups of lattice-ordered groups, Trans. Amer. Math. Soc. 158 (1971), 339-371.

5. A. H. Clifford, A noncommutative ordinally simple linearly ordered group, Proc. Amer. Math. Soc. 2 (1952), 902-903. MR 13, 625.

6. P. F. Conrad, Embedding theorems for abelian groups with valuations, Amer. J. Math. 75 (1953), 1-29. MR 14, 842.

7. P. F. Conrad, J. Harvey and C. Holland, The Hahn embedding theorem for abelian latticeordered groups, Trans. Amer. Math. Soc. 108 (1963), 143-169. MR 27 \#1519.

8. L. Fuchs, Partially ordered algebraic systems, Pergamon Press, New York; AddisonWesley, Reading, Mass., 1963. MR 30 \#2090.

9. ——, Riesz groups, Ann. Scuola Norm. Sup. Pisa (3) 19 (1965), 1-34. MR 31 \#4843.

10. A. M. W. Glass, Which abelian groups can support a directed, interpolation order?, Proc. Amer. Math. Soc. 31 (1972), 395-400.

11. - The lattice of convex directed subgroups of a directed, interpolation group (unpublished paper( $\left.{ }^{1}\right)$ ).

12. P. Hall, On the finiteness of certain soluble groups, Proc. London Math. Soc. (3) 9 (1959), 595-622. MR 22 \#1618.

13. C. Holland, The lattice-ordered group of automorphisms of an ordered set, Michigan Math. J. 10 (1963), 399-408. MR 28 \#1237.

14. P. Jaffard, Sur le spectre d'un groupe réticulé et l'unicité des réalisations irréductibles, Ann. Univ. Lyon. Sect. A (3) 22 (1959), 43-47. MR 22 \#6853.

15. P. Lorenzen, Abstrakte Begründung der multiplikativen Idealtheorie, Math. Z. 45 (1939), 533-553. MR 1, 101.

16. D. Papert, A representation theory for lattice-groups, Proc. London Math. Soc. (3) 12 (1962), 100-120. MR 24 \#A3217.

17. J. R. Teller, A theorem on Riesz groups, Trans. Amer. Math. Soc. 130 (1968), 254-264. MR 37 \#120.

18. E. C. Weinberg, Embedding in a divisible lattice-ordered group, J. London Math. Soc. 42 (1967), 504-506. MR 36 \#91.

Department of Mathematics, University of Wisconsin, Madison, Wisconsin 53706

Department of Mathematics, Bowling Green State University, Bowling Green, OHIO 43402 (Current address)

(1) A copy of this paper is available from the author. It constitutes Chapter 3 of the author's Ph.D. thesis Interpolation groups, University of Wisconsin, Madison, Wisconsin, 1971. 